\title{
Modulator-Controlled Synthesis of Microporous STA-26, an Interpenetrated 8,3- Connected Zirconium MOF with the the-i Topology, and its Reversible Lattice Shift
}

Alice M. Bumstead, ${ }^{1}$ David B. Cordes, ${ }^{1}$ Daniel M. Dawson, ${ }^{1}$, Kristina K. Chakarova, ${ }^{2}$ Mihail Y. Mihaylov, ${ }^{2}$ Claire L. Hobday, ${ }^{3}$ Tina Düren, ${ }^{3}$ Konstantin I. Hadjiivanov, ${ }^{2}$ Alexandra M. Z. Slawin, ${ }^{1}$ Sharon. E. Ashbrook, ${ }^{1}$ Ram R. R. Prasad ${ }^{*, 1}$ and Paul A. Wright*,1

${ }^{1}$ EaStCHEM School of Chemistry, University of St Andrews, Purdie Building, North Haugh, St Andrews KY16 9ST, United Kingdom.

${ }^{2}$ Institute of General and Inorganic Chemistry, Bulgarian Academy of Sciences, Sofia 1113, Bulgaria.

${ }^{3}$ Centre for Advanced Separations Engineering, Department of Chemical Engineering, University of Bath, Bath BA2 7AY, United Kingdom.

*E-mail: rprr@st-andrews.ac.uk; paw2@st-andrews.ac.uk

\begin{abstract}
A fully interpenetrated 8,3-connected zirconium MOF with the the-i topology type, STA-26 (St Andrews porous material-26), has been prepared using the 4,4',4"-(2,4,6trimethylbenzene-1,3,5-triyl)tribenzoate (TMTB) tritopic linker with formic acid as a modulating agent. In the as-prepared form STA-26 possesses Im-3m symmetry compared with the Pm-3m symmetry of the non-interpenetrated analogue, NU-1200, prepared using benzoic acid as a modulator. Upon removal of residual solvent there is a shift between the interpenetrating lattices and a resultant symmetry change to $\mathrm{Cmcm}$ which is fully reversible. This is observed by X-ray diffraction and ${ }^{13} \mathrm{C}$ MAS NMR is also found to be remarkably sensitive to the structural transition. Furthermore, heating STA-26(Zr) in vacuum dehydroxylates the $\mathrm{Zr}_{6}$ nodes leaving coordinatively unsaturated $\mathrm{Zr}^{4+}$ sites, as shown by IR
\end{abstract}


spectroscopy using $\mathrm{CO}$ and $\mathrm{CD}_{3} \mathrm{CN}$ as probe molecules. Nitrogen adsorption at $77 \mathrm{~K}$ together with grand canonical Monte Carlo simulations confirms a microporous, fully interpenetrated, structure with pore volume $0.53 \mathrm{~cm}^{3} \mathrm{~g}^{-1}$ while $\mathrm{CO}_{2}$ adsorption at $196 \mathrm{~K}$ reaches $300 \mathrm{~cm}^{3} \mathrm{STP}$ $\mathrm{g}^{-1}$ at 1 bar. While the pore volume is smaller than that of its non-interpenetrated mesoporous analogue, interpenetration makes the structure more stable to moisture adsorption and introduces shape selectivity in adsorption.

\section{Introduction}

The diverse and growing family of porous metal-organic frameworks (MOFs) ${ }^{[1]}$ constructed from metal-based nodes and organic linkers, offers a range of potential applications in adsorption, ${ }^{[2]}$ sensing,${ }^{[3]}$ catalysis ${ }^{[4]}$ and drug delivery. ${ }^{[5]}$ Among these many materials, the zirconium carboxylates offer some of the most porous and also the most chemically and thermally stable examples. ${ }^{[6]}$ Many of these are based on the $\mathrm{Zr}_{6} \mathrm{O}_{8}$ building block, which may adopt 12-, 10-, 8- or 6-fold coordination with di-, tri- or tetracarboxylate groups of linker molecules, to give structures with $f c u, b c t, c s q$ and $s p n$ topologies.$^{[7]}$ A zirconium carboxylate MOF with the 8,3-connected the topology type, formed with the 4,4',4"-(2,4,6trimethylbenzene-1,3,5-triyl)tribenzoate (TMTB) tritopic linker, was recently reported by two groups and assigned the names $\mathrm{NU}-1200^{[8]}$ and BUT-12. ${ }^{[9]}$ The structure consists of a primitive cubic arrangement of corner-sharing cages with octahedral geometry, each of which possesses a sodalite-like (sod) cage structure (Figure 1). Each 'superoctahedral' cage comprises six $\mathrm{Zr}_{6} \mathrm{O}_{8}$ clusters linked via tritopic TMTB linkers on the faces of the octahedron, and has an internal free diameter of $1.4 \mathrm{~nm}$. Each $\mathrm{Zr}_{6} \mathrm{O}_{8}$ cluster is linked to eight others in a square prismatic arrangement. Charge balance in NU-1200 is reportedly achieved by the addition of hydroxyl groups and water molecules to the zirconium clusters, giving an overall 
framework formula $\mathrm{Zr}_{6}\left(\mu_{3} \mathrm{O}\right)_{4}\left(\mu_{3} \mathrm{OH}\right)_{4}(\mathrm{OH})_{4}\left(\mathrm{OH}_{2}\right)_{4}\left(\mathrm{C}_{30} \mathrm{H}_{24} \mathrm{O}_{6}\right)_{8 / 3} \cdot{ }^{[10,11]}$ Between these 'superoctahedral' cages is a mesoporous pore space, accessible via $2 \mathrm{~nm}$ windows, which is of great interest as a potential host for adsorbates or for species that are catalytically active for the transformation of large molecules.

The reported syntheses of NU-1200 and BUT-12 are similar, but make use of different carboxylic acids as modulators. Modulators have been observed to have a strong effect on MOF crystallisation, controlling particle size and morphology, the density of 'missing linker' defects and even the phase that is formed. ${ }^{[12]}$ Even though the length and flexibility of linkers $^{[13-15]}$ and the influence of solvents ${ }^{[16,17]}$ on regulating the degree of interpenetration has been observed previously, the role of modulators in governing the synthesis of interpenetrated MOFs has not, to the best of our knowledge.

Here we report the role of modulators in controlling the interpenetration in a zirconium MOFs with the the framework topology, and its hafnium analogue. Interpenetration, in which identical lattices have grown one within the other, is observed in many MOFs where their geometry and dimensions allow, and where interactions between the interpenetrating lattices are favourable. ${ }^{[18]}$ The zirconium amino-terephthalate $\mathrm{MOF}$ UiO-66 $\left(\mathrm{NH}_{2}\right)$ and related structural forms with longer ditopic linkers, for example, have been shown to form interpenetrated frameworks, the crystallinity of which can be enhanced by the use of modulators. ${ }^{[14]}$

We designate the interpenetrated Zr-MOF framework STA-26 (St Andrews porous material26) and have characterised its structure, porosity and properties fully. Complementary single crystal X-ray diffraction and $\mathrm{N}_{2}$ and $\mathrm{CO}_{2}$ adsorption (together with molecular simulation of the adsorption) show it is microporous rather than mesoporous; powder diffraction and solid state NMR reveal details of a relative lattice shift upon post-synthetic treatment that modifies 
the window size; IR spectroscopy is used to investigate its adsorption sites; and interpenetration imparts enhanced stability to moisture. Furthermore, we have prepared the hafnium-version of this material, in the knowledge that Hf has similar chemistry in MOF frameworks but that Hf-MOFs can possess properties significantly different from those of their Zr-analogues. ${ }^{[19]}$
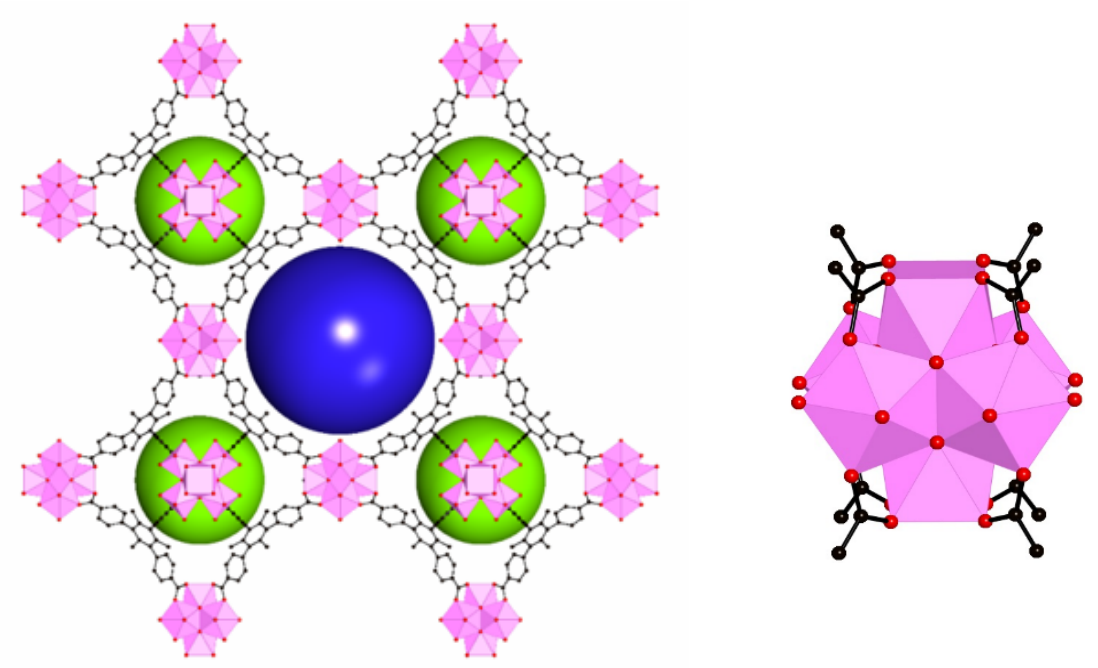

Figure 1 (Left) Polyhedral and ball-stick representation of the structure of NU-1200, which has the the topology type. The mesoporous cages of NU-1200 are shown by the blue sphere while the space within the sodalite-like cages are shown by the green spheres. (Right) The 8connected $\mathrm{Zr}_{6}$ cluster of NU-1200. Hydrogen atoms are omitted for clarity.

\section{Results and Discussion}

A modified NU-1200 synthesis, based on a literature procedure which used benzoic acid as a modulator, gave a material with a PXRD pattern that matched that simulated from the published, non-interpenetrated, structure of NU-1200 in Pm-3m, with unit cell parameter of 28.300 $\AA$ (Figures S1 and S2). ${ }^{[8]}$ Initial synthesis attempts using formic acid as a modulator, 
similar to those reported for the synthesis of BUT-12 $2^{[9]}$ and with a DMF:formic acid molar ratio of 1:2 $(8 \mathrm{ml}: 8 \mathrm{ml})$ gave crystalline solids with a PXRD pattern which could be indexed to a primitive cell with a very similar unit cell parameter to NU-1200 (28.169 ̊) but with strongly different diffraction intensities (Figure 2). Solution-state ${ }^{1} \mathrm{H}$ NMR spectra of the dissolved Zr-MOF showed peaks from the TMTB ligand and also from residual DMF. It was possible to pick out single crystals from these preparations that were sufficiently large for laboratory single crystal diffraction (Figure S3).

After screening different DMF:formic acid molar ratios (Figure S4), it was found that a 1:1.2 molar ratio of DMF:formic acid $(10 \mathrm{ml}: 6 \mathrm{ml})$ gave a microcrystalline material comprising cubic crystals $c a$. 1-2 $\mu \mathrm{m}$ in dimension (Figure S5). In this case the PXRD pattern (Figure 2, right) could be indexed as body-centred cubic, with a similar unit cell to those preparations described above, and this Zr-MOF was designated STA-26(Zr) (STA = St Andrews porous material). Notably, in the absence of formic acid modulator, no crystalline solid forms, whereas adding smaller amounts of formic acid (DMF:formic acid molar ratio 5.8:1) or higher amounts of formic acid (DMF:formic acid 1:2) gives solids for which the PXRD shows considerable intensity in peaks that violate the I-centring that is clearly demonstrated by pure STA-26(Zr) (Figure S4).

A hafnium version, STA-26(Hf), was also prepared with a PXRD pattern that could be indexed to a body-centred cell, unit cell parameter $28.317 \AA$ (Figure 2). 

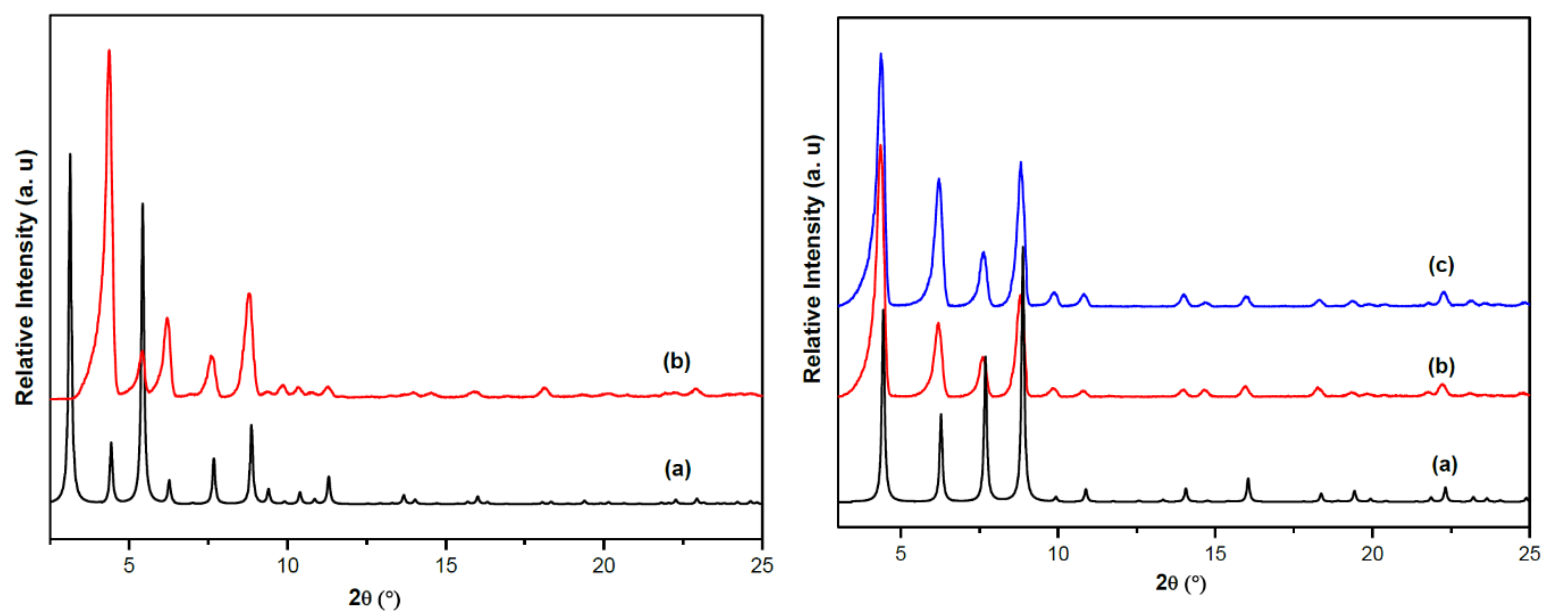

Figure 2 (Left) (a) Simulated PXRD pattern of NU-1200(Zr) compared with (b) that of as-synthesised STA-26(Zr) prepared with DMF:formic acid molar ratio 1:2. (Right) (a) Simulated PXRD of STA$26(\mathrm{Zr})$ compared with those of (b) as-synthesised STA-26(Zr) and (c) as-synthesised STA-26(Hf) (each with a 1:1.2 molar ratio of DMF:formic acid).

SCXRD of a selected crystal of the product from the preparation with a DMF:formic acid molar ratio of 1:2 revealed a structure with cubic symmetry, space group $\mathrm{Im}-3 m$ (Table 1 and Figure 3, left). The PXRD pattern simulated from this structure solution is a close fit to that observed for both $\mathrm{Zr}$ and Hf versions of STA-26 (Figure 2, right), with the small differences in intensity in the low angle peaks attributable to the presence of disordered solvent remaining in the pores. The pattern is very different from that of the non-interpenetrated NU1200, compared directly in Figure 3 (right). This indicates that the I-centred STA-26(Zr) possesses an interpenetrated structure, with two identical lattices of the the topology, each with a structure similar to that of the NU-1200 framework, displaced by a lattice vector $[1 / 2,1 / 2,1 / 2]$. The TMTB linkers exhibit $90^{\circ}$ rotation of the benzoate groups with respect to the central mesitylene core that is characteristic of this linker, and which results in crystallisation of the the 8,3-topology. In STA-26, the sodalite-like cages of one of the interpenetrating frameworks now occupy the mesoporous cavities of the other. 

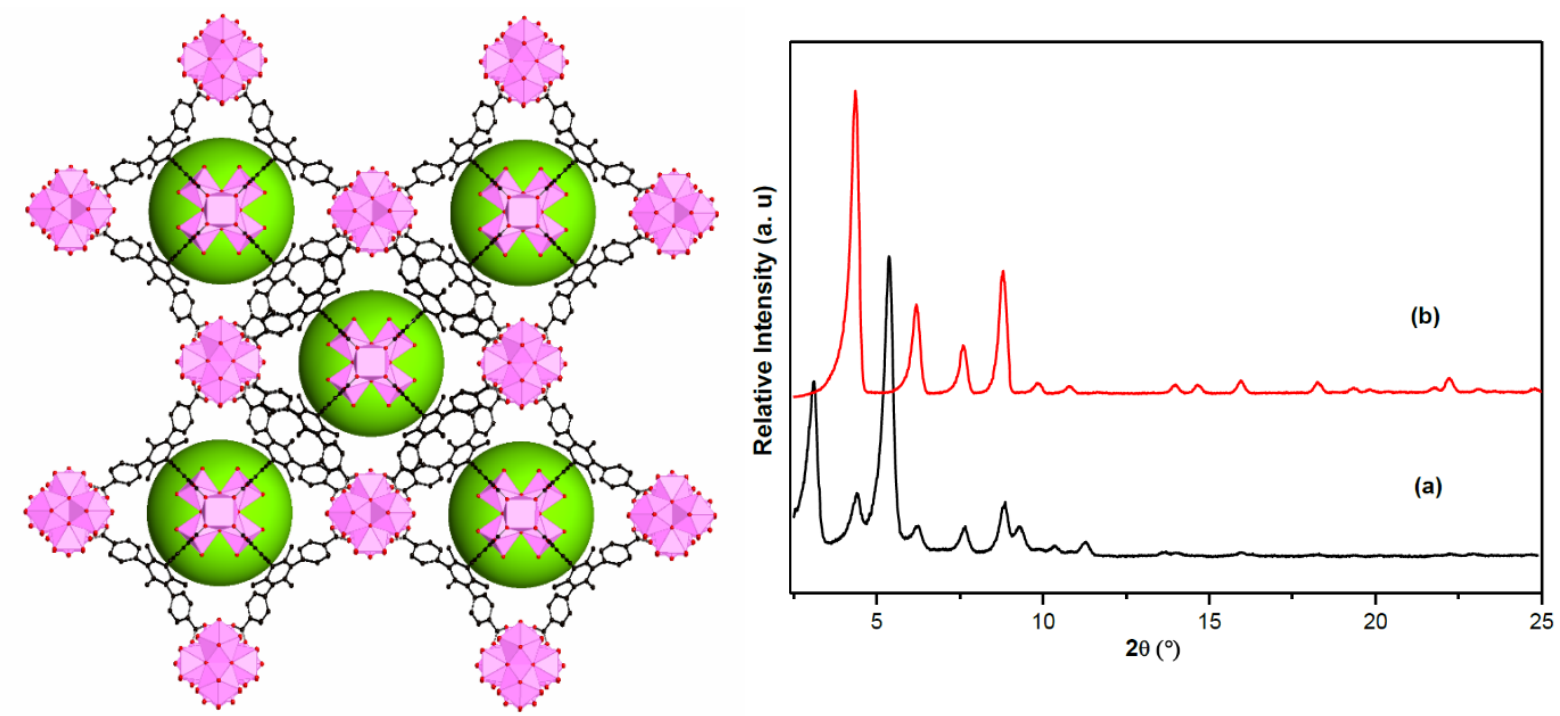

Figure 3. (Left) Structure of STA-26(Zr) with $I m-3 m$ symmetry. It is an interpenetrated analogue of NU-1200, shown in Figure 1. (Right) PXRD patterns of as-synthesised (a) NU-1200(Zr) and (b) STA$26(\mathrm{Zr})$

The linkers of the interpenetrated networks at their closest point display a distance of $5.16 \AA$ between the centres of $\mathrm{C}$ atoms of the adjacent benzene rings. Pore openings with a width of $4.80 \AA$ (once van der Waals radii are taken into account) lead to octahedral cages of internal diameter $14 \AA$ (similar to those of NU-1200). To the best of our knowledge, an interpenetrated $\mathrm{Zr}-\mathrm{MOF}$ with 8-connected $\mathrm{Zr}_{6} \mathrm{O}_{8}$ clusters has not been reported, although it has been shown that by fine tuning the planarity of triangular tetrazolate-based linkers used in the synthesis of copper benzene- or triazine-centered tetrazolates the the and the-i topology can be achieved for 8,3-connected frameworks. ${ }^{[15]}$

Since both NU-1200 and STA-26 have the same overall composition (metal clusters and linkers), the difference in structure must arise from the use of different modulators in the solvothermal preparations. As the formic, benzoic and 2-fluorobenzoic acids have very similar $p$ Ka values $(3.8,4.2 \text { and } 3.8)^{[20]}$ this originates from the different steric effects when attached to the $\mathrm{Zr}_{6}$ clusters during crystal growth. It should be noted that no formate species 
were observed in the digested STA-26 by solution-phase NMR (Figure S6) although benzoic acid was observed in digested NU-1200 (Figure S7).

Although the bulk sample from which the single crystal was taken showed reflections in the powder pattern that violate body centring, prolonged immersion of the bulk ' $1: 2$ ' sample in DMF post-synthesis, followed by drying in air, gave a bulk sample with a body-centred powder pattern similar to that observed for the small crystal ' $1: 1.2$ ' preparation. This suggested that both samples had the same interpenetrated structure, and that the additional peaks derived from a reversible symmetry change related to residual DMF content (Fig. S8).

Table. 1 Crystallographic data for STA-26(Zr) and STA-26(Zr)-C

\begin{tabular}{|c|c|c|}
\hline Label & STA-26(Zr) & STA-26(Zr)- $C$ \\
\hline Formula & $\mathrm{C}_{40} \mathrm{H}_{36} \mathrm{O}_{16} \mathrm{Zr}_{3}$ & $\mathrm{C}_{40} \mathrm{H}_{36} \mathrm{O}_{16} \mathrm{Zr}_{3}$ \\
\hline Formula weight $/ \mathrm{g} \mathrm{mol}^{-1}$ & 1046.35 & 1046.35 \\
\hline Temperature/K & 173 & 173 \\
\hline Crystal system & Cubic & Orthorhombic \\
\hline Space group & $\operatorname{Im} \overline{3} m$ & $\mathrm{Cmcm}$ \\
\hline $\mathrm{a} / \AA$ & $28.16930(14)$ & $40.3620(8)$ \\
\hline $\mathrm{b} / \AA$ & - & $39.8384(8)$ \\
\hline $\mathrm{c} / \AA$ & - & $27.9686(5)$ \\
\hline $\mathrm{V} / \AA$ & $22352.6(3)$ & $44972.3(15)$ \\
\hline Z & 12 & 24 \\
\hline $\mathrm{Q}($ calcd $) \mathrm{g} \mathrm{cm}-3$ & 0.933 & 0.927 \\
\hline Radiation type & $\mathrm{Cu} \mathrm{K} \alpha$ & $\mathrm{Cu} \mathrm{K} \alpha$ \\
\hline$\mu \mathrm{mm}-1$ & 3.725 & 3.703 \\
\hline $\mathrm{F}(000)$ & 6288 & 12576 \\
\hline Rint & 0.1007 & 0.1908 \\
\hline GOF & 1.069 & 1.426 \\
\hline Final R1 values $[\mathrm{I}>2 \sigma(\mathrm{I})]^{\mathrm{a}}$ & 0.0618 & 0.1309 \\
\hline Final wR2 values (all data) ${ }^{\mathrm{b}}$ & 0.1977 & 0.3662 \\
\hline
\end{tabular}




\section{Structural changes upon activation for adsorption measurements}

In order to optimise the accessible pore volume of STA-26(Zr), samples were repeatedly washed with acetone and subsequently dried at $80{ }^{\circ} \mathrm{C}$ prior to measurement of $\mathrm{N}_{2}$ adsorption at $77 \mathrm{~K}$. Under these conditions, the PXRD pattern displays additional reflections that violate the $I$-centring. Subsequent immersion in DMF and drying gave a sample with the original $I$ centred pattern (Figure 4). STA-26(Hf) showed similar behaviour when treated under these conditions (Figure S9). Further, VT-PXRD of STA-26(Zr) shows a loss of I-centring upon heating, which was not reversed on cooling (Figure 5). Taken together, these observations suggest that traces of residual DMF solvent keep the interpenetrated lattices apart, whereas their removal results in a structural change that was reversible by re-introduction of DMF.

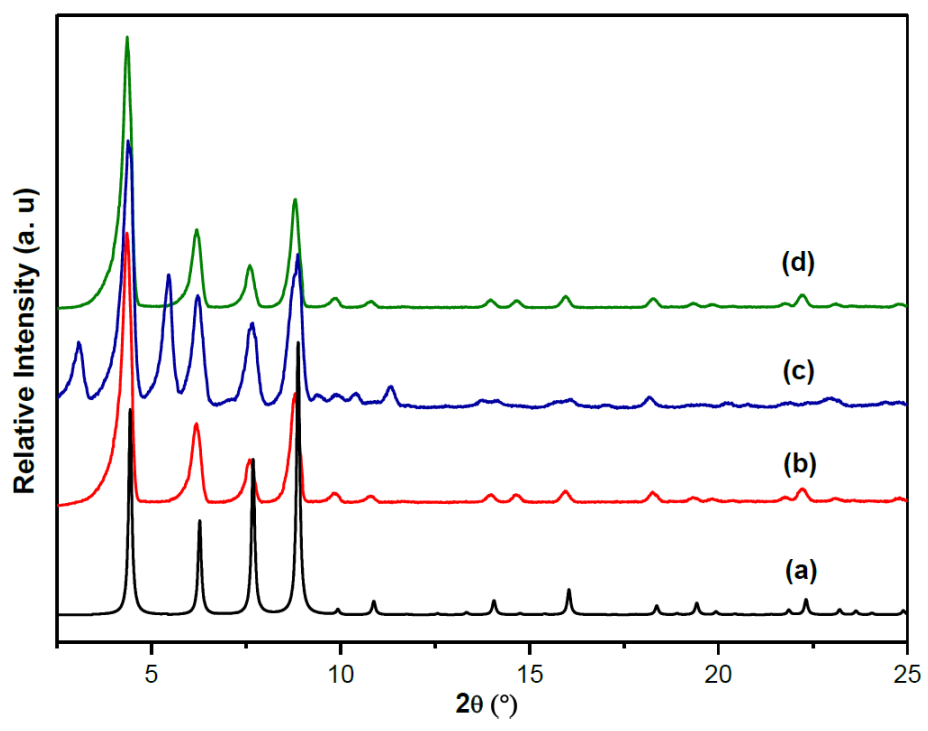

Figure 4 Comparison of (a) the simulated PXRD pattern for STA-26(Zr) with those of (b) assynthesised STA-26(Zr) (1:1.2 prep.), (c) STA-26(Zr) after 1 week in acetone with solvent exchange every $24 \mathrm{~h}$, followed by drying, (d) after immersion in DMF and drying. 


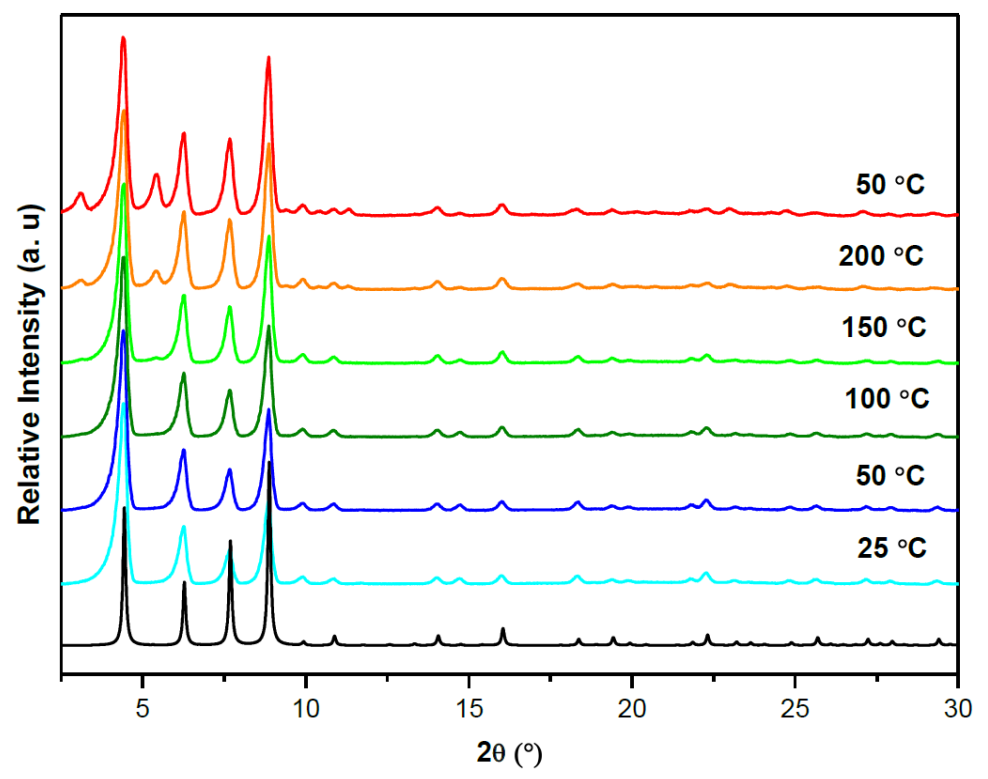

Figure 5 VT-PXRD of as-synthesised STA-26(Zr) heated from $25{ }^{\circ} \mathrm{C}$ to $200{ }^{\circ} \mathrm{C}$ and then cooled to $50{ }^{\circ} \mathrm{C}$, compared with simulated pattern for as-synthesised STA-26(Zr), the black curve.

To investigate this further, SCXRD was performed on a single crystal of as-synthesised STA$26(\mathrm{Zr})$ that had been kept in acetone for a week with the solvent replaced every $24 \mathrm{~h}$ and subsequently dried at $80{ }^{\circ} \mathrm{C}$. The structure was solved in the orthorhombic Cmcm space group, and is here referred to as STA-26(Zr)- $C$ (See Table 1 and the cif file in the ESI for crystallographic details). There has been a relative movement of one lattice with respect to the other, changing the distance between the TMTB linkers and the $\mathrm{Zr}_{6} \mathrm{O}_{8}$ clusters of the two frameworks (Figure 6). Whereas in body-centred STA-26(Zr) the distance between the central phenyl ring of the TMTB of adjacent linkers is the same in every case $(4.74 \AA)$, in STA-26(Zr)-C there are three different distances, $3.571 \AA$, $4.74 \AA$ and $7.41 \AA$. Consequently, STA-26(Zr)- $C$ possesses three different pore openings with free diameters $3.57 \AA, 4.43 \AA$ and $5.24 \AA$ leading to cages of unchanged internal diameter $(14 \AA)$. This relative movement of the interpenetrating frameworks changes the overall symmetry of the structure, so that instead of 
a single crystallographic environment for the linkers in STA-26(Zr), there are now three different sets of linker environments in STA-26(Zr)- $C$.

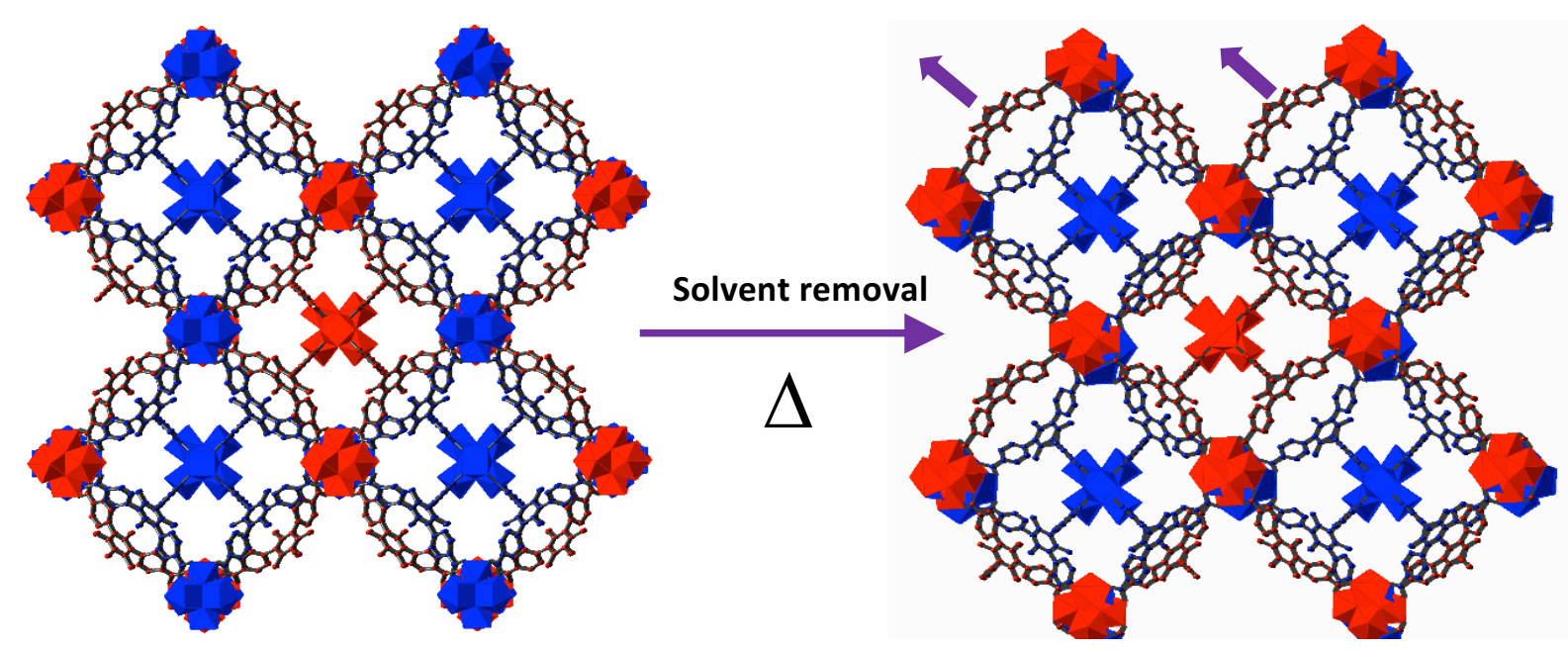

Figure 6 Structural change of (left) STA-26(Zr) $(\mathrm{Im}-3 \mathrm{~m})$ to (right) STA-26(Zr)-C (Cmcm) upon removal of residual solvent molecules. The relative direction of lattice movement of one of the interpenetrated lattices (with clusters in red) is denoted by purple arrows.

Owing to its sensitivity to small changes in the local structure, solid-state ${ }^{13} \mathrm{C}$ NMR spectroscopy can be used to follow this structural transition. For body-centred STA-26(Zr), each of the chemically-distinct carbon atoms of the TMTB linker structure gives a single resonance, as expected from the symmetry (Figures 7 and S10, and S11 for the Hf-form). Notably, the spectrum is similar to that observed for the non-interpenetrated NU-1200 (Figure S12), in which the ligands are all crystallographically equivalent, but with small changes in chemical shift. In the ${ }^{13} \mathrm{C}$ CP MAS NMR spectrum of STA-26(Zr)- $C$ prepared by solvent removal, each of the signals shows splittings that indicate the presence of distinct crystallographic environments, due to the relative movement of the frameworks. As an example, whereas there is only one crystallographically distinct $\mathrm{CH}_{3}$ in the I-centred structure, there are eight in the C-centred structure, giving rise to the observed splitting in the resonance at $16-18 \mathrm{ppm}$. 
When the desolvated structure is reimmersed in DMF, the splittings of the spectral resonances disappear and the spectrum is similar to that of the original STA-26(Zr) (Figure 7). This confirms that the $I$-centred structure is stabilised by the introduction of solvent molecules residing in the pores. If these are removed either by continuous solvent exchange and/or heating, one lattice moves with respect to each other to achieve a lower energy position.

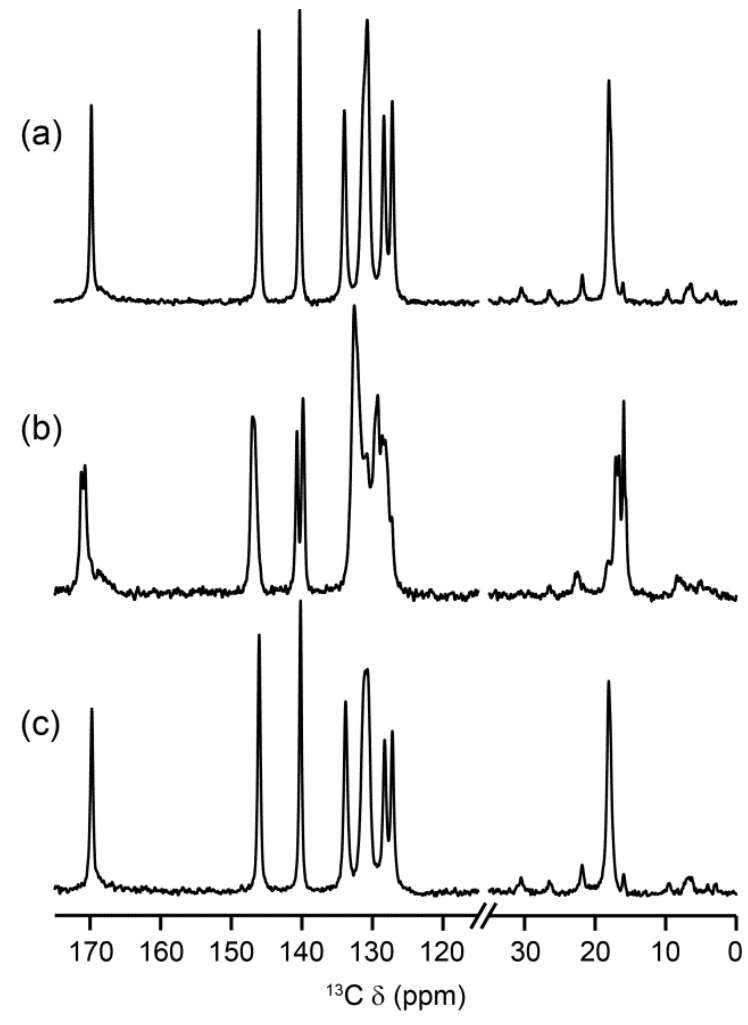

Figure $7{ }^{13} \mathrm{C}$ CP MAS NMR spectra of (a) as-synthesised STA-26(Zr), (b) STA-26(Zr)- $C$ obtained after prolonged solvent exchange with acetone and (c) after reimmersion in DMF.

It is notable that as-synthesised materials showing PXRD peaks of relatively low intensity violating the $I$-centring also show splittings in the ${ }^{13} \mathrm{C}$ CP MAS NMR spectrum, although these are not as large as when washed and dried at $80{ }^{\circ} \mathrm{C}$ (Figure S14). It is likely that this is 
a result of intermediate stages of framework movement where the structure contains different levels of DMF.

Furthermore, the effect of immersion of the C-form of STA-26 in other solvent molecules (Figure S13) showed that while DMF caused full return to I symmetry, tetrahydrofuran had no effect while the bulkier $\mathrm{N}, \mathrm{N}$-dibutyl- and $\mathrm{N}, \mathrm{N}$-diethylformamide strongly reduced the intensities of reflections attributed to the 'off-centring' of the second lattice. This indicates solvent polarity plays a controlling role in this effect.

VT-PXRD of STA-26(Zr)- $C$ showed that the lattice-shifted structure is stable and no further symmetry changes occur up to $200{ }^{\circ} \mathrm{C}$ (Figure 8 ). The overall crystallinity of the structure is retained after the experiment and when cooled down to room temperature, and there is now very close agreement between observed solvent-free STA-26(Zr)- $C$ and the pattern simulated from the crystal structure.

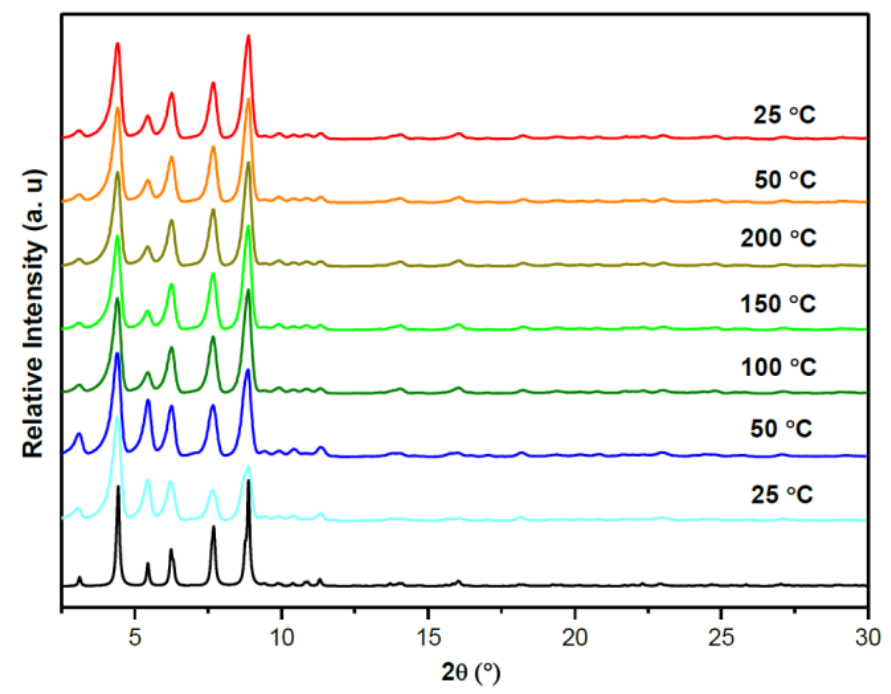

Figure 8. VT-PXRD patterns of STA-26(Zr)-C from $25{ }^{\circ} \mathrm{C}$ to $200{ }^{\circ} \mathrm{C}$ and then cooled down to $50{ }^{\circ} \mathrm{C}$ and $25{ }^{\circ} \mathrm{C}$ with simulated pattern of STA-26(Zr)- $C$. 
TGA of as-prepared samples of STA-26(Zr) (Figure S15) indicates an initial weight loss of $8 \%$ corresponding to the removal of adsorbed volatile solvent molecules residing in the pores and another loss of $8 \%$ around $150-300{ }^{\circ} \mathrm{C}$, which can be attributed to the dehydroxylation of the $\mathrm{Zr}_{6}$ clusters and removal of residual DMF. The structure decomposes above $400{ }^{\circ} \mathrm{C}$.

Figure 9 shows the IR spectra of STA-26, as-prepared and evacuated at room temperature. The two strong bands at 1600 and $1406 \mathrm{~cm}^{-1}$ are assigned to coordinated carboxylates. ${ }^{[21]}$ The as-prepared sample is characterized by a broad band at $3400 \mathrm{~cm}^{-1}$ due to adsorbed water. Evacuation at RT removes this band completely, indicating weak water adsorption. Therefore, the sample treated in this way has few strong adsorption sites such as coordinately unsaturated $\mathrm{Zr}$ sites associated with missing-linker defects.
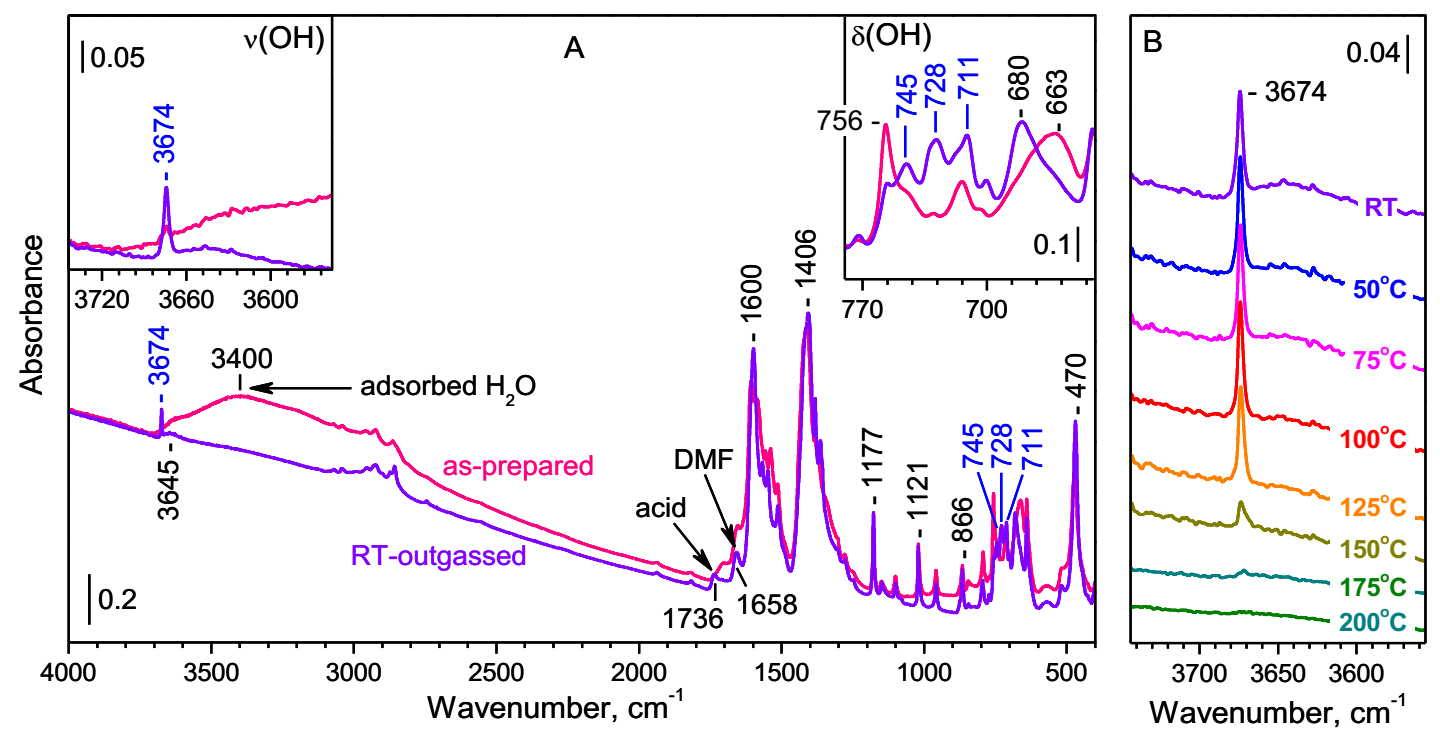

Figure 9. (a) IR spectra of (left) STA-26(Zr) as-prepared (pink) and evacuated at room temperature (violet) and (b) IR spectra showing the dehydroxylation of STA-26(Zr).

Water desorption leads to development of a sharp band in the $\mathrm{OH}$ stretch region at $3674 \mathrm{~cm}^{-1}$ due to isolated $\mathrm{OH}$ groups attached to zirconium oxide clusters. ${ }^{[1-23]}$ For the related $\mathrm{Zr}$ MOF 
NU-1000, this band was attributed to include vibrations of terminal and bridging $\mathrm{OH}$ groups ${ }^{[23]}$ Surprisingly, three deformation $\mathrm{OH}$ bands at 745,729 and $711 \mathrm{~cm}^{-1}$ also appeared, but deuteration of the sample (see Figure S16) produced one OD deformation band. This revealed that the three $\mathrm{OH}$ bands are not indicative of heterogeneity of $\mathrm{OH}$ groups but rather are a consequence of a spectroscopic effect arising from mixing of $\mathrm{OH}$ deformation modes with other MOF vibrations. These $\mathrm{OH}$ groups are of low acidity as the water $\mathrm{H}$-bonded to them can be easily removed. A weak and broad feature around $3645 \mathrm{~cm}^{-1}$ is probably due to a small fraction of weakly $\mathrm{H}$-bonded hydroxyls $\mathrm{OH}$ groups affected by strongly bound adsorbates. Residual DMF $\left(1659 \mathrm{~cm}^{-1}\right)$ and free acid $\left(1736 \mathrm{~cm}^{-1}\right)$ are observed for the room temperature evacuated sample. The DMF is removed upon outgassing at $175^{\circ} \mathrm{C}$.

The stability of intra-framework hydroxyls was studied upon stepwise heating in vacuum. The dehydroxylation of STA-26(Zr) sample starts at $100{ }^{\circ} \mathrm{C}$, proceeds gradually with heating, and is completed at $200{ }^{\circ} \mathrm{C}$ (Figure 9). This process was found to be reversible. The dehydroxylation route can be described as condensation of hydroxyl groups: $2 \mathrm{OH} \rightarrow \mathrm{O}^{2-}+$ $\mathrm{H}_{2} \mathrm{O}$. This is in accordance with the TGA of STA-26(Zr). The picture is similar to that observed for NU-1200 and is attributed to the loss of water and hydroxyl groups from the $\mathrm{Zr}_{6} \mathrm{O}_{8}$ clusters. ${ }^{[10,11]}$

To investigate changes in the internal surface chemistry of STA-26(Zr) upon heating, CO, $\mathrm{CD}_{3} \mathrm{CN}$ and $\mathrm{CO}_{2}$ were used as probe molecules in an extended IR spectroscopic study. The adsorption of probe molecules was examined for both hydroxylated and dehydroxylated forms of STA-26(Zr).

Surface acidity was assessed by adsorption of $\mathrm{CO}$ and $\mathrm{CD}_{3} \mathrm{CN}$ at cryogenic and ambient temperature, respectively (Figure 10). With the hydroxylated sample both probes reveal the existence of weak Brønsted acidity which is typical of MOF materials. ${ }^{[24,25]}$ Adsorption of CO 
leads to development of an $\mathrm{OH} \cdots \mathrm{CO}$ band at $2149 \mathrm{~cm}^{-1}$ (Figure 10A). Simultaneously, a red shift of the $\mathrm{OH}$ stretching band of $68 \mathrm{~cm}^{-1}$ is detected (note that the largest such shift reported with MOF materials is $90 \mathrm{~cm}^{-1[26]}$ ). The $\mathrm{OH} \cdots \mathrm{NCCD}_{3}$ complexes were detected at $2273 \mathrm{~cm}^{-1}$.

The bands associated with $\mathrm{OH} \cdots \mathrm{CO}$ and $\mathrm{OH}^{\cdots} \cdot \mathrm{NCCD}_{3}$ species appeared with strongly reduced intensities on the dehydroxylated sample. However, in this case, bands indicative of the presence of open $\mathrm{Zr}^{4+}$ sites were detected $\left(\mathrm{Zr}^{4+} \ldots \mathrm{CO}\right.$ at $2170 \mathrm{~cm}^{-1}$ and $\mathrm{Zr}^{4+} \ldots \mathrm{NCCD}_{3}$ at 2300 $\mathrm{cm}^{-1}$, see Figs. 10B and D, respectively). Note that, as a strong base, $\mathrm{CD}_{3} \mathrm{CN}$ can displace water adsorbed on Lewis acid sites and can thus detect some open $\mathrm{Zr}^{4+}$ sites that are not visible by $\mathrm{CO}$ as a probe. However, it appears that the concentration of such sites is negligible, as indicated by the very weak feature around $2300 \mathrm{~cm}^{-1}$ in Figure $10 \mathrm{C}$. These results rule out the existence in our sample of a large fraction of clusters terminated by water ligands only. 

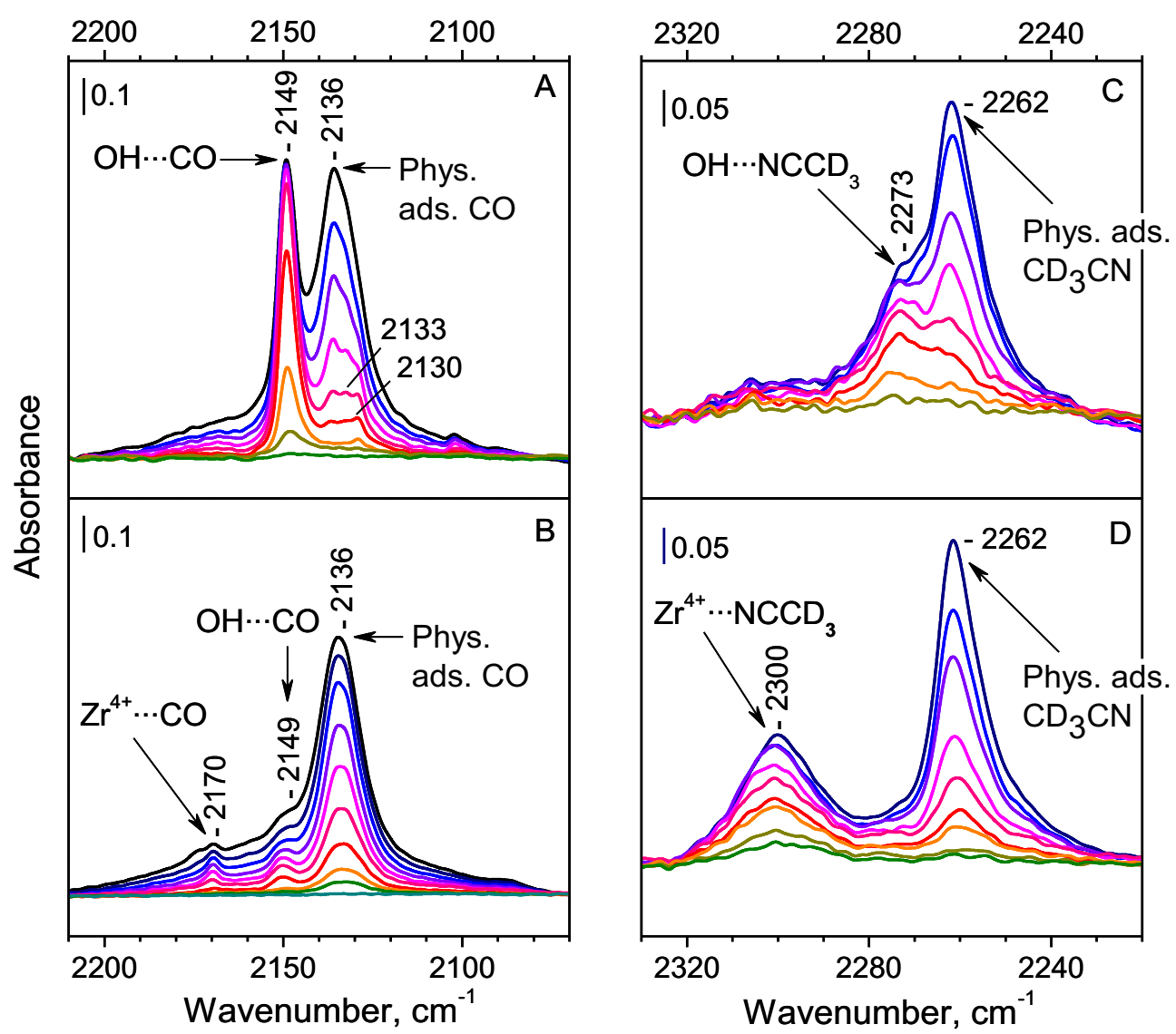

Figure 10. (A, B) IR spectra of $\mathrm{CO}$ adsorbed at $100 \mathrm{~K}$ and $(\mathrm{C}, \mathrm{D}) \mathrm{CD}_{3} \mathrm{CN}$ adsorbed at room temperature on STA-26(Zr) sample evacuated at room temperature (upper panels) and at $175^{\circ} \mathrm{C}$ (bottom panels), with spectra from continually increasing doses of the IR probe gases represented in green to blue.

Spectra of adsorbed $\mathrm{CO}_{2}$ were acquired at room temperature with $\mathrm{CO}_{2}$ equilibrium pressure varied in the 1 - 50 mbar range. The $\mathrm{v}_{\mathrm{as}}\left(\mathrm{CO}_{2}\right)$ bands obtained with the dehydroxylated STA26(Zr) sample (Figure 11B) are less than one-half the intensity of the hydroxylated material (Figure 11A).This indicates that the $\mathrm{OH}$ groups can play an important role as adsorption sites for $\mathrm{CO}_{2}$. The second derivatives of the spectra (shown in the left insets) reveal that the main band consists of two components. The high frequency component can be related to $\mathrm{CO}_{2}$ adsorbed on structural $\mathrm{OH}$ groups $(\mathrm{OH}-\mathrm{OCO})$ because (i) it is hardly observed with the 
highly dehydroxylated sample and (ii) it correlates with the shift of the $\mathrm{OH}$ stretching band (see the right inset in Figure 11A). The low frequency component can be attributed to weakly and non-specifically adsorbed $\mathrm{CO}_{2}$.
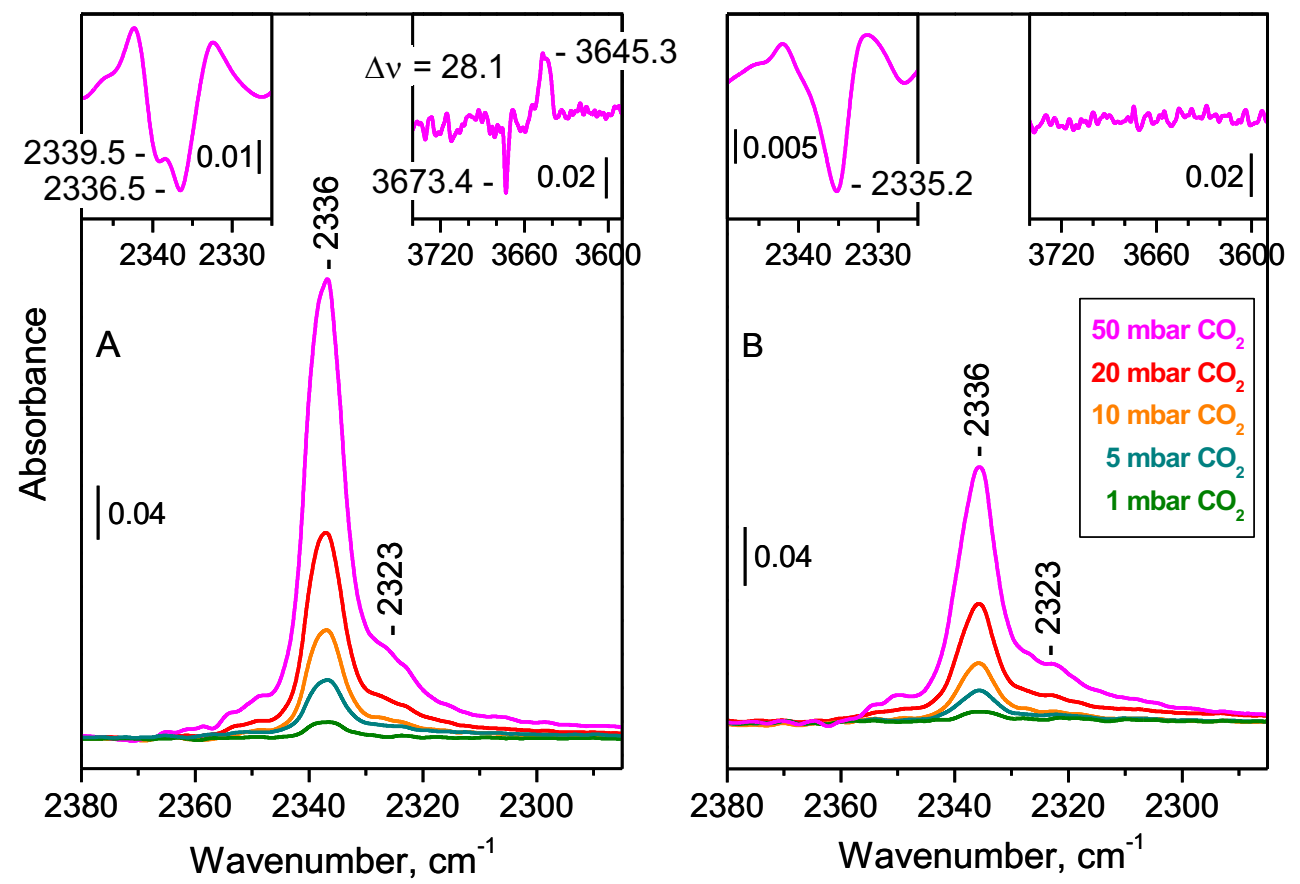

Figure 11. (A) IR spectra of $\mathrm{CO}_{2}$ adsorption of STA-26(Zr) evacuated at room temperature and (B) at $150{ }^{\circ} \mathrm{C}$.

The pore structure of STA-26 was probed by $\mathrm{N}_{2}$ and $\mathrm{CO}_{2}$ adsorption measurements, after an activation protocol that, according to our IR and PXRD studies, dehydroxylates the clusters and results in conversion to the STA-26(Zr)- $C$ form. $\mathrm{N}_{2}$ adsorption at $77 \mathrm{~K}$ gives a Type I isotherm typical of a microporous solid (Figure 12, left). This contrasts strongly with that reported for $\mathrm{NU}-1200^{[8]}$ and the isotherm measured for the NU-1200 prepared in this work (Figure S17), both of which show a Type IV isotherm typical of a mesoporous structure. 

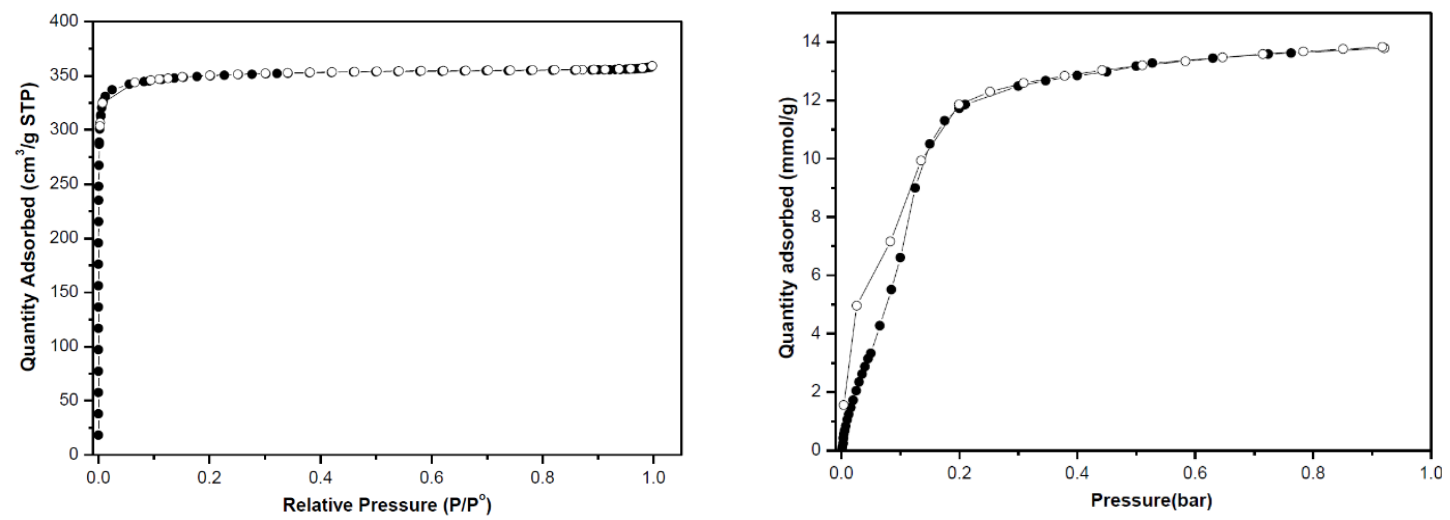

Figure 12. (Left) $\mathrm{N}_{2}$ adsorption isotherm at $77 \mathrm{~K}$ and (right) $\mathrm{CO}_{2}$ adsorption isotherm at $196 \mathrm{~K}$ measured for STA-26(Zr) activated at $150{ }^{\circ} \mathrm{C}$ for $16 \mathrm{~h}$ (closed symbols), with desorption (open symbols).

Simulated nitrogen adsorption isotherms in STA-26(Zr)-C and NU-1200 (Figure 13) show reasonable agreement with the experimentally measured isotherms, although the total pore volume is higher than that measured, presumably because not all the pore space is accessible, or there is some loss of structure. For STA-26, around $75 \%$ of the calculated value is observed. Note that the lattice shift ( $I$ to $C$ transition) has only a very minor effect on the simulated adsorption isotherm.

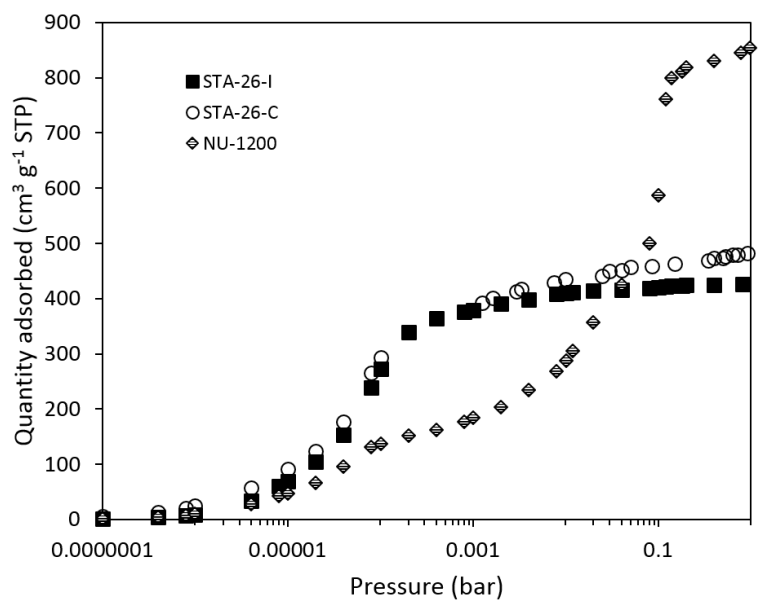

Figure 13. Simulated $\mathrm{N}_{2}$ adsorption isotherms for STA-26(Zr)-I, STA-26(Zr)- $C$ and NU1200 at $77 \mathrm{~K}$. 
These results emphasise that, while non-interpenetrated NU-1200 is mesoporous, with pore volume deriving both from the space within the sod cages and the mesoporous space between them, in STA-26 the mesoporous cavities of one the framework are occupied by sod cages of the other, giving a microporous material. This confirms our interpretation of the single crystal XRD, which might without the $\mathrm{N}_{2}$ adsorption data also be interpreted as a partially interpenetrated structure, with each of the lattices having the same fractional occupancy. Such a structure would possess some mesoporosity and so show an inflexion in the $\mathrm{N}_{2}$ adsorption isotherm.

$\mathrm{CO}_{2}$ adsorption measured at $196 \mathrm{~K}$ approaches $300 \mathrm{~cm}^{3} \mathrm{STP} \mathrm{g}^{-1}, 1$ bar (Figure 12 right), and at $298 \mathrm{~K}$ reaches $160 \mathrm{~cm}^{3} \mathrm{STP} \mathrm{g}^{-1}$ at 10 bar (Figure S18), in each case showing an inflection at around $80 \mathrm{~cm}^{3}$ STP $\mathrm{g}^{-1}$. GCMC simulations of the adsorption isotherms of $\mathrm{CO} 2$ on STA26(Zr) and NU-1200 at $196 \mathrm{~K}$ are shown in Figure S19. Comparison with the observed isotherm confirms that STA-26 is fully interpenetrated and microporous. The simulations also yield detailed information about the guest-framework interaction and the location of the guest molecules. Figure 14 shows histograms of the simulated $\mathrm{CO}_{2} / \mathrm{MOF}$ interaction energies at 1 bar. While NU-1200 has some low energy sites (i.e. strong interaction sites) mainly around the $\mathrm{Zr}$-nodes of the framework (Figure 14d), the adsorption behaviour is dominated by weak adsorption sites (interaction energies $\sim-2 \mathrm{~kJ} \mathrm{~mol}^{-1}$ ) in the centre of the octahedral pores. In STA-26(Zr)- $C$ the Zr-clusters act as strong adsorption sites, and furthermore interpenetration in STA-26(Zr)-C results in smaller pore sizes and stronger adsorption sites (Figure 13a and c). Simulated adsorption surfaces of $\mathrm{CO}_{2}$ at 0.01 bar on STA-26(Zr)- $I$ and $C$ show that the lattice shift gives rise to an additional adsorption site in the void between phenyl groups of neighbouring interpenetrating nets (Figure S20). 

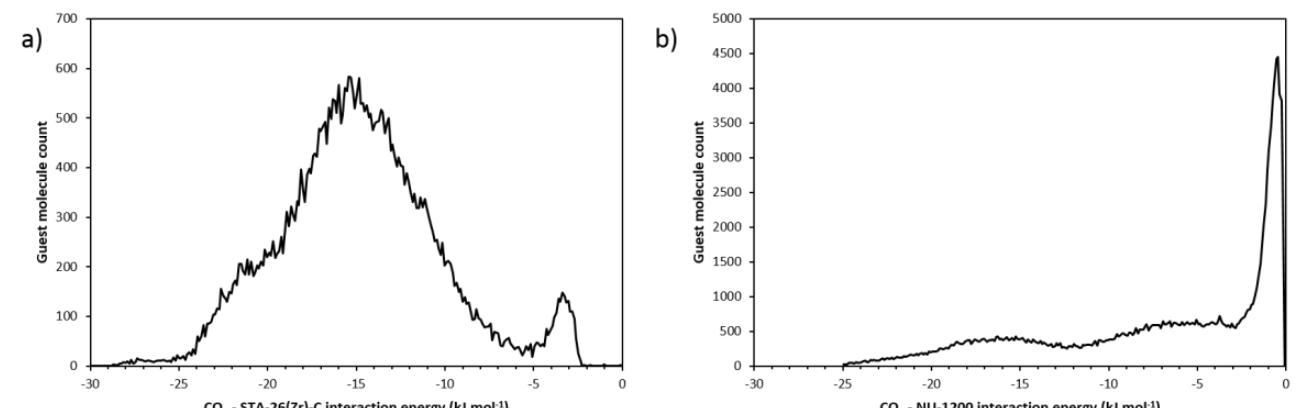

c)
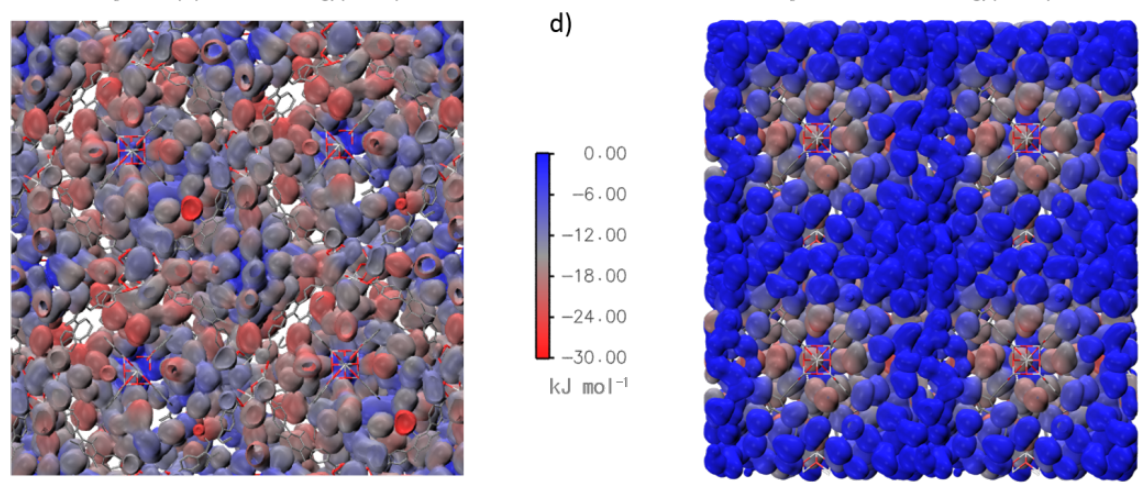

Figure 14. Energy density distributions of $\mathrm{CO}_{2} / \mathrm{MOF}$ interactions in (a) STA-26(Zr)- $C$ and (b) NU1200 at $196 \mathrm{~K}$ and 1 bar. (c) and (d) Surface plots of the $\mathrm{CO}_{2} / \mathrm{MOF}$ interaction energy, where blue $=0$ $\mathrm{kJ} \mathrm{mol}^{-1}$ and red $=-30 \mathrm{~kJ} \mathrm{~mol}^{-1}$.

To demonstrate the difference in window size between microporous STA-26(Zr) and mesoporous NU-1200, dye uptake and catalytic behaviour of the MOF were also studied. Following the previous dye uptake studies performed by Lin et al., ${ }^{[27]}$ NU-1200 and STA-26 were kept in ethanolic solutions of Fuchsin basic dye for $10 \mathrm{~h}$, followed by subsequent filtration and multiple washes with ethanol. Mesoporous pore openings enable NU-1200 to achieve higher levels of dye uptake in comparison with STA-26(Zr), as clearly indicated by the difference in intensity of colour (Figure 15).
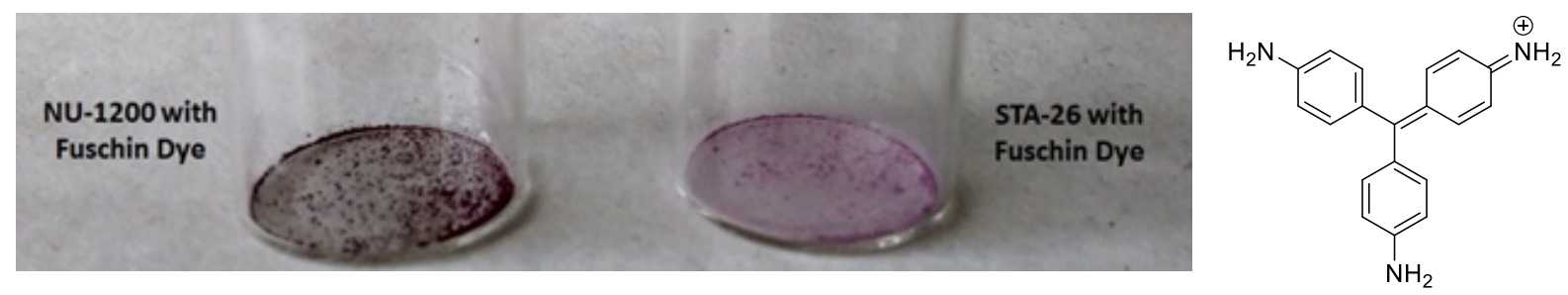

Figure 15. NU-1200 and STA-26(Zr) after exposure to a solution of Fuchsin basic dye (right). 
Furthermore, in the Lewis acid-catalysed condensation of 4'-fluoroacetophenone and benzylamine (Scheme S1), STA-26(Zr) gave only $28 \%$ conversion towards the imine, in comparison with $51 \%$ for the NU-1200 (Figure S21). As both MOFs possess similar $\mathrm{Zr}_{6} \mathrm{O}_{8}$ clusters, the higher conversion rates of NU-1200 are attributed to the free movement of the large reactant and product molecules within its mesopores, while the lower observed conversion over microporous STA-26 could result from surface catalysis.

Thermal and water stability of MOFs are two major concerns that limit their application. While the thermal stability has been steadily improved ${ }^{[7,28]}$ the water instability of MOFs remains a significant limitation. One cause of instability, giving rise to extremely low surface areas after activation from water, is the high capillary forces acting on the walls of pores containing water. ${ }^{[29]}$ Consequently, MOFs are best activated after being kept in low boiling point solvents or in supercritical $\mathrm{CO}_{2} \cdot{ }^{[30]}$ As interpenetration has been reported to increase the overall stability of MOFs, ${ }^{[13]}$ we tested the water stability of STA-26(Zr). Following the protocol by Farha et al., as-prepared STA-26(Zr) was kept in water for $48 \mathrm{~h}$ and subsequently activated at $100{ }^{\circ} \mathrm{C} \cdot{ }^{[31]}$ PXRD showed peaks corresponding to change in symmetry and can be indexed to STA-26(Zr) showing the I to C symmetry change. The overall crystallinity of the MOF was well-preserved under these conditions (Figure 16). By contrast, when NU-1200 was kept in water and activated under similar conditions, some broadening of reflections occurred. Furthermore, at $77 \mathrm{~K}$, the $\mathrm{N}_{2}$ adsorption isotherm of NU-1200 activated directly from water showed significantly lower porosity, while STA-26(Zr) retained its porous nature. This indicates that the interpenetrated geometry of STA-26 enhances its stability to water. 

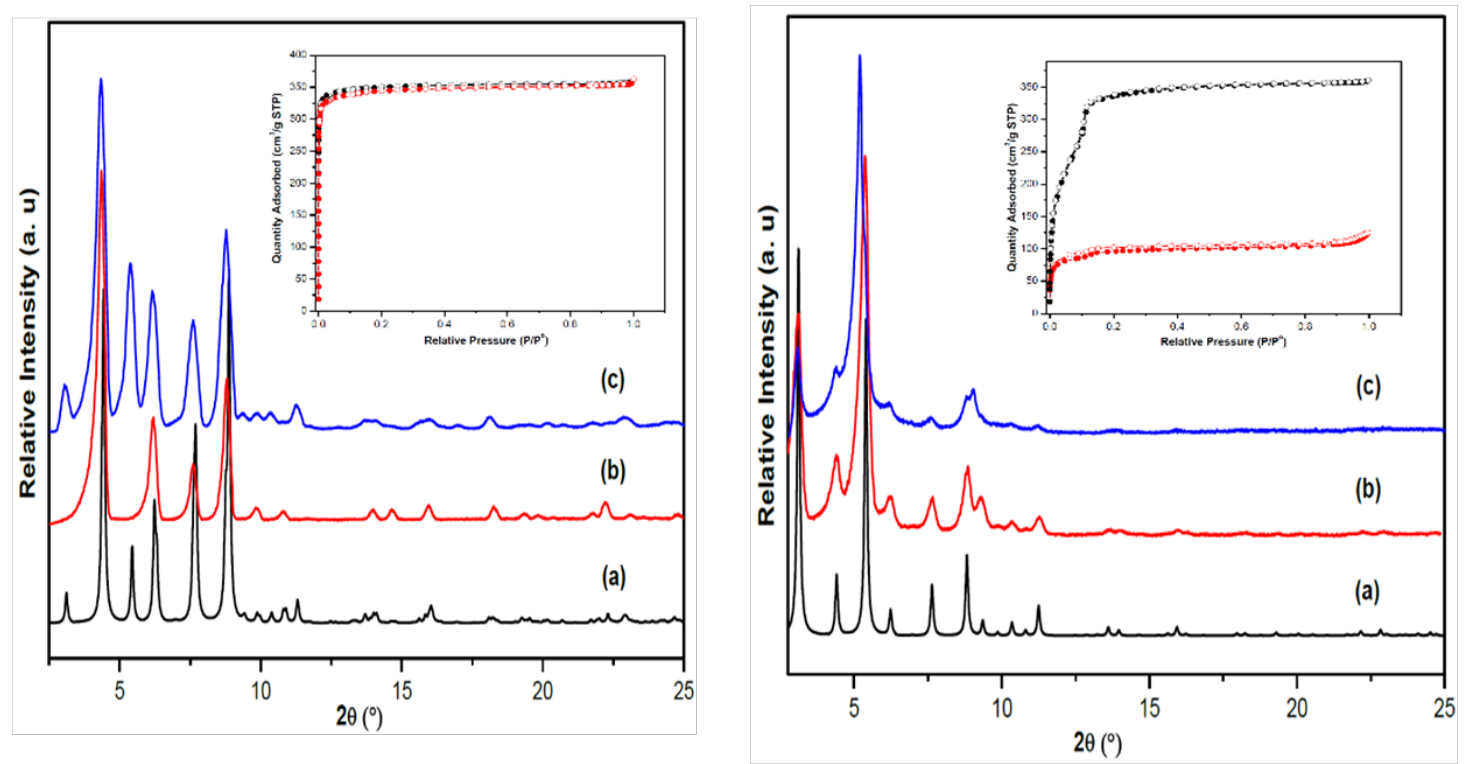

Figure 16. Left, (a) Simulated PXRD pattern for STA-26(Zr)-C, (b) as-prepared STA-26(Zr), (c) STA-26(Zr) after $48 \mathrm{~h}$ water stability test and direct activation from water. Inset: $\mathrm{N}_{2}$ isotherm of STA26(Zr) activated at $150{ }^{\circ} \mathrm{C}$ after solvent exchange with acetone (black) and directly activated from water (red). Right, (a) simulated PXRD pattern for NU-1200, (b) as-prepared NU-1200, (c) NU-1200 after water stability test and direct activation from water. Inset: $\mathrm{N}_{2}$ isotherm of NU-1200 activated at $150^{\circ} \mathrm{C}$ after solvent exchange with acetone (black) and directly activated from water (red).

\section{Conclusions}

A route has been devised to a fully interpenetrated version of the Zr-based MOF, NU-1200, which we denote STA-26(Zr). The Hf-analogue of STA-26 has also been prepared. As in NU-1200, the TMTB linker in STA-26 shows the characteristic geometry, where the presence of the methyl groups on the central benzene ring of the linker results in rotation of the benzoate groups and consequently favours the the topology type with eight-connected $\mathrm{Zr}_{6} \mathrm{O}_{8}$ clusters. In the STA-26 structure, two lattices with the the topology type interpenetrate.

Whether the interpenetrated or non-interpenetrated form crystallises depends on the modulating agent used in our syntheses. Use of benzoic acid gives NU-1200 whereas in the presence of formic acid the interpenetrated structure results. It is likely that during 
crystallisation the observed presence of benzoate groups bound to the clusters sterically inhibits the growth of a second, interpenetrating, lattice.

Upon removal of solvent from STA-26, there is a shift in the position of one interpenetrating lattice with respect to the other, resulting in a symmetry change from $I m-3 m$ to $C m c m$, which is reversible upon reimmersion in DMF (the synthesis solvent). The post-synthetic structural changes are observed not only by X-ray diffraction, but also, remarkably, by solid-state ${ }^{13} \mathrm{C}$ NMR, which shows peak splittings as the symmetry changes. Heating at $125^{\circ} \mathrm{C}$ and above results in dehydroxylation of the cluster and IR spectroscopy using Lewis basic probe molecules reveals the presence of Lewis acid sites, likely to be coordinatively unsaturated $\mathrm{Zr}^{4+}$ cations.

Activated samples of STA-26 give $\mathrm{N}_{2}$ adsorption $77 \mathrm{~K}$ isotherms of Type I, confirming the microporous nature of the STA-26 structure, compared to the Type IV isotherm of the mesoporous non-interpenetrated analogue NU-1200. $\mathrm{CO}_{2}$ adsorption shows gradual pore filling at $196 \mathrm{~K}$ up to ca. $0.2 \mathrm{bar}$, which is well described by grand canonical Monte Carlo simulations of adsorption on the microporous interpenetrated structure. While the microporosity and especially the reduced window size of ca. $5 \AA$ will make STA-26 unfavourable for the adsorption and catalytic transformations of large molecules, it possesses good porosity for $\mathrm{N}_{2}$ and $\mathrm{CO}_{2}$ even after direct activation from water, demonstrating that it is a stable and size-selective adsorbent. 


\section{Experimental Section}

Materials and Methods The synthesis of TMTB, 4,4',4''-(2,4,6-trimethylbenzene-1,3,5triyl)tribenzoic acid, was performed by modifying previously reported methods.$^{[8,9,32]} \mathrm{A}$ detailed experimental procedure is provided in the Supporting Information. NU-1200(Zr) was synthesised by modifying the published work by Liu et al ${ }^{[8]} \mathrm{ZrCl}_{4}$ (48 $\mathrm{mg}, 0.20 \mathrm{mmol}$ ), TMTB (40 mg, $0.08 \mathrm{mmol})$ and benzoic acid/2-fluorobenzoic acid (1.40/1.61 g, $11.5 \mathrm{mmol})$ were ultrasonically dissolved in DMF ( $8 \mathrm{~mL})$ in a pyrex vial. The solution was heated at 120 ${ }^{\circ} \mathrm{C}$ for $48 \mathrm{~h}$ in an oven before being cooled to room temperature. The supernatant solution was extracted with a syringe and the resulting white solid was washed multiple times with DMF followed by acetone and dried in an oven at $80^{\circ} \mathrm{C}(26.0 \mathrm{mg})$. Single crystals of STA26(Zr) were prepared using a reaction procedure similar to that of Wang et al for the synthesis of BUT-12. ${ }^{[9]} \mathrm{ZrCl}_{4}(48 \mathrm{mg}, 0.20 \mathrm{mmol}$ ) and TMTB (40 mg, $0.08 \mathrm{mmol}$ ) were ultrasonically dissolved in a solvent mixture of DMF $(8 \mathrm{~mL})$ and formic acid $(8 \mathrm{~mL})$ in a 20 $\mathrm{mL}$ Pyrex vial. The resulting solution was gradually heated to $120{ }^{\circ} \mathrm{C}$ with a ramp rate of 3 ${ }^{\circ} \mathrm{C} \min ^{-1}$ and kept there for $48 \mathrm{~h}$ before being cooled to room temperature. The supernatant solution was removed and the colourless crystals obtained were washed multiple times with DMF followed by acetone, then dried in an oven at $80{ }^{\circ} \mathrm{C}(35.9 \mathrm{mg})$.

For the synthesis of single phase STA-26(Zr), $\mathrm{ZrCl}_{4}(48 \mathrm{mg}, 0.2 \mathrm{mmol})$ and TMTB $(40 \mathrm{mg}$, $0.08 \mathrm{mmol})$ were ultrasonically dissolved in a solvent mixture of DMF (10 mL) and formic acid $(6 \mathrm{~mL})$ in a $20 \mathrm{~mL}$ Pyrex vial. The resulting solution was gradually heated to $120{ }^{\circ} \mathrm{C}$ with a ramp rate of $3{ }^{\circ} \mathrm{C} \min ^{-1}$ and kept there for $48 \mathrm{~h}$ before being cooled to room temperature. The supernatant solution was removed and the resulting white solid was washed multiple times DMF followed by acetone, then dried in an oven at $80{ }^{\circ} \mathrm{C}(53.0 \mathrm{mg})$. The related STA-26(Hf) was synthesised using $\mathrm{HfCl}_{4}(64.1 \mathrm{mg}, 0.20 \mathrm{mmol})$ and TMTB (40 mg, 
$0.08 \mathrm{mmol})$, ultrasonically dissolved in a solvent mixture of DMF (10 $\mathrm{mL})$ and formic acid (6 $\mathrm{mL}$ ) in a Pyrex vial. The resulting solution was gradually heated to $120^{\circ} \mathrm{C}$ with a ramp rate of $3{ }^{\circ} \mathrm{C} \min ^{-1}$ and kept there for $48 \mathrm{~h}$ before being cooled to room temperature. The supernatant solution was removed and the white solid product was washed multiple times with DMF followed by acetone and dried in an oven at $80{ }^{\circ} \mathrm{C}(55.3 \mathrm{mg})$.

Materials and methods Powder X-ray diffraction (PXRD) patterns of finely ground powder were collected in Debye-Scherrer geometry from Stoe STAD i/p diffractometers with primary monochromation $\left(\mathrm{Cu} \mathrm{K}_{\alpha 1}, \lambda=1.54056 \AA\right)$, using $0.7 \mathrm{~mm}$ glass capillaries. Variable temperature PXRD (VT-PXRD) measurements were performed in air, with a ramp rate of 5 ${ }^{\circ} \mathrm{C} \min ^{-1}$ and held at the desired temperature for 10 min prior to measurements. Data were collected from 25 to $200{ }^{\circ} \mathrm{C}$ and after cooling down to $25{ }^{\circ} \mathrm{C}$ for STA-26(Zr) and STA26(Zr)- $C$, using a Cobra Plus non-liquid-nitrogen cryostream (Oxford Cryosystems).

For solution-state nuclear magnetic resonance (NMR) spectra of the MOF samples, the protocol developed by Lin et. $a l^{[33]}$ was used. Prior to analysis, $10 \mathrm{mg}$ of MOF in DMSO- $\mathrm{d}_{6}$ $(0.5 \mathrm{~mL})$ was added to a saturated solution of $\mathrm{K}_{3} \mathrm{PO}_{4}$ in $\mathrm{D}_{2} \mathrm{O}(0.5 \mathrm{~mL})$ and stirred at room temperature until the MOF was completely dissolved.

Solid-state NMR spectra were recorded using a Bruker Avance III spectrometer equipped with a 9.4 T superconducting magnet (Larmor frequencies of $400.1 \mathrm{MHz}$ for ${ }^{1} \mathrm{H}$ and 100.6 $\mathrm{MHz}$ for ${ }^{13} \mathrm{C}$ ). For the ${ }^{13} \mathrm{C}$ NMR spectra, samples were packed into standard $\mathrm{ZrO}_{2}$ rotors with an outer diameter of $4 \mathrm{~mm}$ and rotated at the magic angle at a rate of $12.5 \mathrm{kHz}$. Spectra were recorded with cross polarisation $(\mathrm{CP})$ from ${ }^{1} \mathrm{H}$ with a contact pulse (ramped for ${ }^{1} \mathrm{H}$ ) of $2 \mathrm{~ms}$. Signal averaging was carried out for between 1024 and 19968 transients with a recycle interval of $3 \mathrm{~s}$. Two-pulse phase modulation (TPPM) decoupling of ${ }^{1} \mathrm{H}\left(v_{1} \approx 100 \mathrm{kHz}\right)$ was 
carried out during acquisition. For the ${ }^{1} \mathrm{H}$ NMR spectra, samples were packed into standard $\mathrm{ZrO}_{2}$ rotors with an outer diameter of $1.9 \mathrm{~mm}$ and rotated at the magic angle at a rate of 40 $\mathrm{kHz}$. Spectra were recorded using a rotor-synchronised spin-echo pulse sequence with an echo delay of $25 \mu$ s. Signal averaging was carried out for 64 or 128 transients with a recycle interval of $3 \mathrm{~s}$. Chemical shifts are reported in ppm relative to TMS, using L-alanine as a secondary solid reference $\left(\mathrm{CH}_{3} \delta=20.5 \mathrm{ppm}\right.$ and $\left.\mathrm{NH}_{3} \delta=8.5 \mathrm{ppm}\right)$.

Thermogravimetric analysis (TGA) of all samples were carried out on a Netzsch TGA 760 for a temperature range of $20-900{ }^{\circ} \mathrm{C}$ at a heating rate of $5^{\circ} \mathrm{C} \mathrm{min}{ }^{-1}$ in a continuous air flow. $\mathrm{N}_{2}$ adsorption isotherms for STA-26(Zr) and NU-1200 samples were measured volumetrically on a Micrometrics Tristar. The $\mathrm{CO}_{2}$ adsorptions isotherms were measured gravimetrically on Hiden IGA porosimeter. All samples were activated at $150{ }^{\circ} \mathrm{C}$ for $16 \mathrm{~h}$ prior to measurements unless otherwise stated.

The IR spectra of STA-26(Zr) on $\mathrm{KBr}$ pellet were recorded using a Nicolet Avatar 360 spectrometer (DTGS detector) and the spectra of a self-supporting STA-26(Zr) pellet were recorded using a Nicolet 6700 FTIR spectrometer (MCT detector). During the experiments, up to 128 scans were accumulated at a spectral resolution of $2 \mathrm{~cm}^{-1}$ with an accuracy of 0.01 $\mathrm{m}^{-1}$. Specially designed IR cells were used for the experiments. One cell, equipped with $\mathrm{CaF}_{2}$ windows, permitted the measurement of spectra at low $(100 \mathrm{~K})$ and ambient temperatures. Another, equipped with $\mathrm{KBr}$ windows, allowed the measurement of spectra at a pre-set temperature in the range between 100 and $300 \mathrm{~K}$. The cells were directly connected to a vacuum-adsorption apparatus with a residual pressure lower than $10^{-3} \mathrm{~Pa}$.

For catalytic studies, both NU-1200 and STA-26(Zr) were activated at $150{ }^{\circ} \mathrm{C}$ for $16 \mathrm{~h}$ under vacuum. In the test imine formation, 4'-fluoroacetophenone $(1 \mathrm{mmol})$ and benzylamine (1 mmol) in dry, degassed toluene (5 mL) were added to the activated MOF (2 mol\%, $42 \mathrm{mg}$ ) 
and heated with stirring at $90{ }^{\circ} \mathrm{C}$ for $24 \mathrm{~h}$ under argon. After completion of the reaction, the conversion rates were determined by ${ }^{19} \mathrm{~F}$ NMR.

\section{Crystal Structure determination}

Single crystal X-ray diffraction (SCXRD) data for body centred STA-26(Zr), here STA26(Zr)-I, and STA-26(Zr)-C were collected at $173 \mathrm{~K}$ using a Rigaku MM-007HF High brilliance RA generator/confocal optics and Rigaku XtaLAB P100 system, with $\mathrm{Cu} \mathrm{K}_{\alpha}$ radiation $(\lambda=1.54187 \AA)$. Intensity data for all compounds were collected using both $\omega$ and $\phi$ steps, accumulating area detector images spanning at least a hemisphere of reciprocal space. All data were corrected for Lorentz polarization effects. A multiscan absorption correction was applied by using CrysAlisPro. ${ }^{[34]}$ Structures were solved by dual-space methods (SHELXT) ${ }^{[35]}$ and refined by full-matrix least-squares against $\mathrm{F}^{2}$ (SHELXL2016/6) $\cdot{ }^{[36]}$ Non-hydrogen atoms were refined anisotropically, and alkyl and aryl hydrogen atoms were refined using a riding model. Water hydrogens in STA-26(Zr) were located from the difference map and refined subject to distance and angle restraints. Similar water hydrogens could not be located for STA-26(Zr)-C. All calculations were performed using the CrystalStructure interface. ${ }^{[37]}$ The data for STA-26(Zr)-C showed non-merohedral twinning (twin law - $0.503-0.51 .014-0.487-0.5-1.0140 .487-0.50 .014$, twin fraction $15.5 \%$ ), which was accounted for in the refinement, and also showed signs that several further minor twins might also be present; although attempts to refine the data taking these into account did not improve structure quality. Both structures showed large voids, $12119 \AA^{3}$ for STA-26(Zr)I (54.2\% of unit cell volume) and $25893 \AA^{3}$ for STA-26(Zr)-C (57.6\% of unit cell volume) and the SQUEEZE ${ }^{[38]}$ routine implemented in PLATON ${ }^{[39]}$ was used to remove the contribution of the disordered electron density in the void spaces. The crystallographic data (excluding structure factors) of the structures reported in this paper have been deposited into 
the Cambridge Crystallographic Data Centre with CCDC Numbers 1571655 for STA-26(Zr)I and 1571656 for STA-26(Zr)-C.

\section{Simulation details}

Gas adsorption was simulated using grand canonical Monte Carlo (GCMC) simulations, implemented in the multipurpose code MuSiC. ${ }^{[40]}$ Framework structures were taken from measured or reported crystallographic cif files. The structures were assumed to have dehydrated $\mathrm{Zr}_{6} \mathrm{O}_{8}$ cores. In addition, disordered $\mathrm{H}$-atoms on the $\mathrm{CH}_{3}$ groups were ordered for the initial optimisation of the structures. This lowered the symmetry of STA-26(Zr) from Im$3 m$ to $I 432$ and STA-26(Zr)-C from $C m c m$ to $C 222$. Due to the $\mu$ VT ensemble imposed in GCMC the framework atoms were fixed in the optimised positions. For each gas $\left(\mathrm{CO}_{2}\right.$ and $\mathrm{N}_{2}$ ) at each pressure, $1 \times 10^{9}$ Monte Carlo steps were performed where each step consists of either a random translation, insertion or deletion, and random rotation - all equally weighted. The first $40 \%$ of the steps were used for equilibration and the remaining used to calculate the ensemble averages. Standard Lennard-Jones (LJ) potentials were used to model the dispersive interactions between the framework and gases. The Trappe force field was used to model $\mathrm{CO}_{2}$, and $\mathrm{N}_{2} \cdot{ }^{[41]}$ Coulombic interactions were included and calculated using Wolf Coulombic summations which are much more efficient than Ewald summations. ${ }^{[42]}$ Interactions beyond $18 \AA$ were neglected. To calculate the gas-phase fugacity the Peng-Robinson equation of state was used. ${ }^{[43]}$

Acknowledgements We acknowledge the support of the EPSRC/St Andrews Criticat CDT (RRRP, PAW) and the European Community Seventh Framework Program (FP7/20072013) number 608490] (project $\left.\mathrm{M}_{4} \mathrm{CO}_{2}\right)(\mathrm{KKC}, \mathrm{MYM}, \mathrm{KIH}, \mathrm{PAW})$. SEA would like to thank 
the Royal Society and Wolfson Foundation for a merit award. This research made use of the Balena High Performance Computing (HPC) Service at the University of Bath. The research data (and/or materials) supporting this publication can be accessed at DOI: XXX.

\section{Electronic Supplementary Information}

Supporting information is available on additional NMR and IR spectra, PXRD, electron and optical micrographs, and measured and calculated adsorption isotherms .

\section{References}

1. (a) H. Furukawa, K. E. Cordova, M. O'Keeffe, O. M. Yaghi, Science 2013, 341, 1230444;

(b) H.-C. Zhou, J. R. Long, O. M. Yaghi, Chem. Rev. 2012, 112, 673-674.

(2) (a) K. Sumida, D. L. Rogow, J. A. Mason, T. M. McDonald, E. D. Bloch, Z. R. Herm, T. H. Bae, J. R. Long, Chem. Rev. 2012, 112, 724-781. (b) J. Li, R. Kuppler, H. Zhou, Chem. Soc. Rev. 2009, 38, 1477-1504.

(3) (a) L. E. Kreno, K. Leong, O. K. Farha, M. Allendorf, R. P. Van Duyne, J. T. Hupp, Chem. Rev. 2012, 112, 1105-1125. (b) M. G. Campbell, S. F. Liu, T. M. Swager, M. Dincă, J. Am. Chem. Soc. 2015, 137, 13780-13783.

(4) (a) J. Liu, L. Chen, H. Cui, J. Zhang, L. Zhang, C.-Y. Su, Chem. Soc. Rev. 2014, 43, 6011-6061. (b) L. Ma, C. Abney, W. Lin, Chem. Soc. Rev. 2009, 38, 1248-1256. (c) J. Jiang, O. M. Yaghi, Chem. Rev. 2015, 115, 6966-6997. (c) M. Rimoldi, A. J. Howarth, M. R. DeStefano, S. Goswami, P. Li, J. T. Hupp, O. K. Farha, ACS Catal. 2017, 7, 997-1014.

(5) P. Horcajada, R. Gref, T. Baati, P. K. Allan, G. Maurin, P. Couvreur, G. Ferey, R. E. Morris, C. Serre, Chem. Rev. 2012, 112, 1232-1268.

(6) (a) J. H. Cavka, S. Jakobsen, U. Olsbye, N. Guillou, C. Lamberti, S. Bordiga, K. P. Lillerud, J. Am. Chem. Soc. 2008, 6, 13850-13851. (b) D. Feng, K. Wang, J. Su, T. F. Liu, J. 
Park, Z. Wei, M. Bosch, A. Yakovenko, X. Zou, H. C. Zhou, Angew. Chem., Int. Ed. 2015, 54, 149-154. (c) V. Bon, I. Senkovska, I. A. Baburin, S. Kaskel, Cryst. Growth Des. 2013, $13,1231-1237$.

(7) Y. Bai, Y. Dou, L.-H. Xie, W. Rutledge, J.-R. Li, H.-C. Zhou, Chem. Soc. Rev. 2016, 45, 2327-2367.

(8) T. F. Liu, N. A. Vermeulen, A. J. Howarth, P. Li, A. A. Sarjeant, J. T. Hupp, O. K. Farha, Eur. J. Inorg. Chem. 2016, 27, 4266.

(9) B. Wang, X. L. Lv, D. Feng, L. H. Xie, J. Zhang, M. Li, Y. Xie, J. R. Li, H. C. Zhou, J. Am. Chem. Soc. 2016, 138, 6204-6216.

(10) N. Planas, J. E. Mondloch, S. Tussupbayev, J. Borycz, L. Gagliardi, J. T. Hupp, O. K. Farha, C. J. Cramer, J. Phys. Chem. Lett. 2014, 5, 3716-3723.

(11) D. Yang, V. Bernales, T. Islamoglu, O. K. Farha, J. T. Hupp, C. J. Cramer, L. Gagliardi, B. C. Gates, J. Am. Chem. Soc. 2016, 138, 15189-15196.

(12) (a) A. Umemura, S. Diring, S. Furukawa, H. Uehara, T. Tsuruoka, S. Kitagawa, J. Am. Chem. Soc. 2011, 133, 15506-15513. (b) S. Diring, S. Furukawa, Y. Takashima, T. Tsuruoka, S. Kitagawa, Chem. Mater. 2010, 22, 4531-4538. (c) G. C. Shearer, S. Chavan, S. Bordiga, S. Svelle, U. Olsbye, K. P. Lillerud, Chem. Mater. 2016, 28, 3749-3761. (d) G. C. Shearer, J. G. Vitillo, S. Bordiga, S. Svelle, U. Olsbye, K. P. Lillerud, Chem. Mater. 2016, 28, 7190-7193. (e) V. Bon, I. Senkovska, M. S. Weiss, S. Kaskel, CrystEngComm, 2013, 15, 9572-9577.

(13) (a) O. K. Farha, C. D. Malliakas, M. G. Kanatzidis, J. T. Hupp, J. Am. Chem. Soc. 2010, 132, 950-952. (b) A. Schaate, P. Roy, T. Preuße, S. J. Lohmeier, A. Godt, P. Behrens, Chem. - A Eur. J. 2011, 17, 9320-9325.

(14) J. Lippke, B. Brosent, T. Von Zons, E. Virmani, S. Lilienthal, T. Preuße, M. Hulsmann, A. M. Schneider, S. Wuttke, P. Behrens, A. Godt, Inorg. Chem. 2017, 56, 748-761.

(15) M. Dincă, A. Dailly, C. Tsay, J. R. Long, Inorg. Chem. 2008, 47, 11-13.

(16) S. B. Choi, H. Furukawa, H. J. Nam, D. Y. Jung, Y. H. Jhon, A. Walton, D. Book, M. O’Keeffe, O. M Yaghi, J. Kim, Angew. Chem., Int. Ed. 2012, 51, 8791-8795. 
(17) A. Ferguson, L. Liu, S. J. Tapperwijn, D. Perl, F.-X. Coudert, S. Van Cleuvenbergen, T. Verbiest, M. A. van der Veen, S. G. Telfer, Nat. Chem. 2016, 8, 250-257.

(18) H.-L. Jiang, T. A. Makal, H.-C. Zhou, Coordination Chemistry Reviews. 2013, 257, 2232-2249.

(19) (a) V. Bon, I. Senkovska, I. A. Baburin, S. Kaskel, Cryst. Growth Des. 2013, 13, 12311237. (b) D. Feng, H. -L. Jong, Y. -P. Chen, Z. -Y. Gu, Z. Wei, H. -C. Zhou, Inorg. Chem. 2013, 52, 12661-12667. (c) M. H. Beyzavi, R. C. Klet, S. Tussupbayev, J. Borycz, N. A. Vermeulen, C. J. Cramer, J. F. Stoddart, J. T. Hupp, O. K. Farha, J. Am. Chem. Soc. 2014, 136, 15861-15864. (d) M. Rimoldi, A. J. Howarth, M. R. DeStefano, L. Lin, S. Goswami, P. Li, J. T. Hupp, O. K. Farha, ACS Catal, 2017, 7, 997-1014.

(20) D. H. Ripin and D. E. Evans, pKa's of Inorganic and Oxo-Acids. http:// evans.harvard.edu/pdf/evans_pKa_table.pdf.

(21) L. Valenzano, B. Civalleri, S. Chavan, S. Bordiga, M. H. Nilsen, S. Jakobsen, K. P. Lillerud, C. Lamberti, Chem. Mater. 2011, 23, 1700-1718.

(22) A. D. Wiersum, E. Soubeyrand-Lenoir, Q. Yang, B. Moulin, V. Guillerm, M. B. Yahia, S. Bourrelly, A. Vimont, S. Miller, C. Vagner, M. Daturi, G. Clet, C. Serre, G. Maurin, P. L. Llewellyn, Chem. Asian J. 2011, 6, 3270-3280.

(23) N. Planas, J. E. Mondloch, S. Tussupbayev, J. Borycz, L. Gagliardi, J. T. Hupp, O. K. Farha, C. J. Cramer, J. Phys. Chem. Lett. 2014, 5, 3716-3723.

(24) K. Hadjiivanov, Adv. Catal., 2014, 57, 99-318.

(25) M. Mihaylov, S. Andonova, K. Chakarova, A. Vimont, E. Ivanova, N. Drenchev, K. Hadjiivanov, Phys. Chem. Chem. Phys., 2015, 17, 24304-24314.

(26) A. Vimont, H. Leclerc, F. Mauge, M. Daturi, J.-C. Lavalley, S. Surble, C. Serre, G. Férey, J. Phys. Chem. C. 2007, 111, 383-388.

(27) L. Ma, M. J. Falkowski, C. Abney, W. Lin, Nat. Chem. 2010, 2, 838-846.

(28) A. J. Howarth, Y. Liu, P. Li, Z. Li, T. C. Wang, J. T. Hupp, O. K. Farha, Nat. Rev. Mater. 2016, 53, 15018, 15018. 
(29) J. E. Mondloch, M. J. Katz, N. Planas, D. Semrouni, L. Gagliardi, J. T. Hupp, O. K. Farha, Chem. Commun. 2014, 50, 8944-8946.

(30) A. P. Nelson, O. K. Farha, K. L. Mulfort, J. T. Hupp, J. Am. Chem. Soc. 2009, 131, 458460.

(31) A. J. Howarth, A. W. Peters, N. A. Vermeulen, T. C. Wang, J. T. Hupp, O. K. Farha, Chem. Mater. 2017, 29, 26-39.

(32) F. Carson, E. Martínez-Castro, R. Marcos, G. G. Miera, K. Jansson, X. Zou, B. MartínMatute, Chem. Commun. 2015, 51, 10864-10867.

(33) N. C. Thacker, Z. Lin, T. Zhang, J. C. Gilhula, C. W. Abney, W. Lin, J. Am. Chem. Soc. 2016, 138, 3501-3509.

(34) CrysAlisPro v1.171.38.43i. Rigaku Oxford Diffraction, Rigaku Corporation, Oxford, U.K. 2015.

(35) G. M. Sheldrick, Crystal structure refinement with SHELXL. Acta Crystallogr. Sect. A. 2015, $71,3-8$

(36) G. M. Sheldrick, Crystal structure refinement with SHELXL. Acta Crystallogr. Sect. C. 2015, 71, 3-8.

(37) CrystalStructure v4.2. Rigaku Americas, The Woodlands, Texas, USA, and Rigaku Corporation, Tokyo, Japan, 2013.

(38) A. L. Spek, Acta Crystallogr. Sect C. 2015, 71, 9-18.

(39) A. L. Spek, Acta Crystallogr. Sect D. 2009, 65, 148-155.

(40) A. Gupta, S. Chempath, M. J. Sanborn, L. A. Clark, R. Q. Snurr, Mol. Simul., 2003, 29, $29-46$.

(41) J. J. Potoff, J. I. Siepmann, AIChE J, 2001, 47, 1676-1682.

(42) D. Wolf, P. Keblinski, S. R. Phillpot, J. Eggebrecht, J. Chem. Phys., 1999, 110, 82548282.

(43) D. Peng, D. B. Robinson, Ind Eng Chem Fund, 1976, 15, 59-64. 
Graphical Abstract

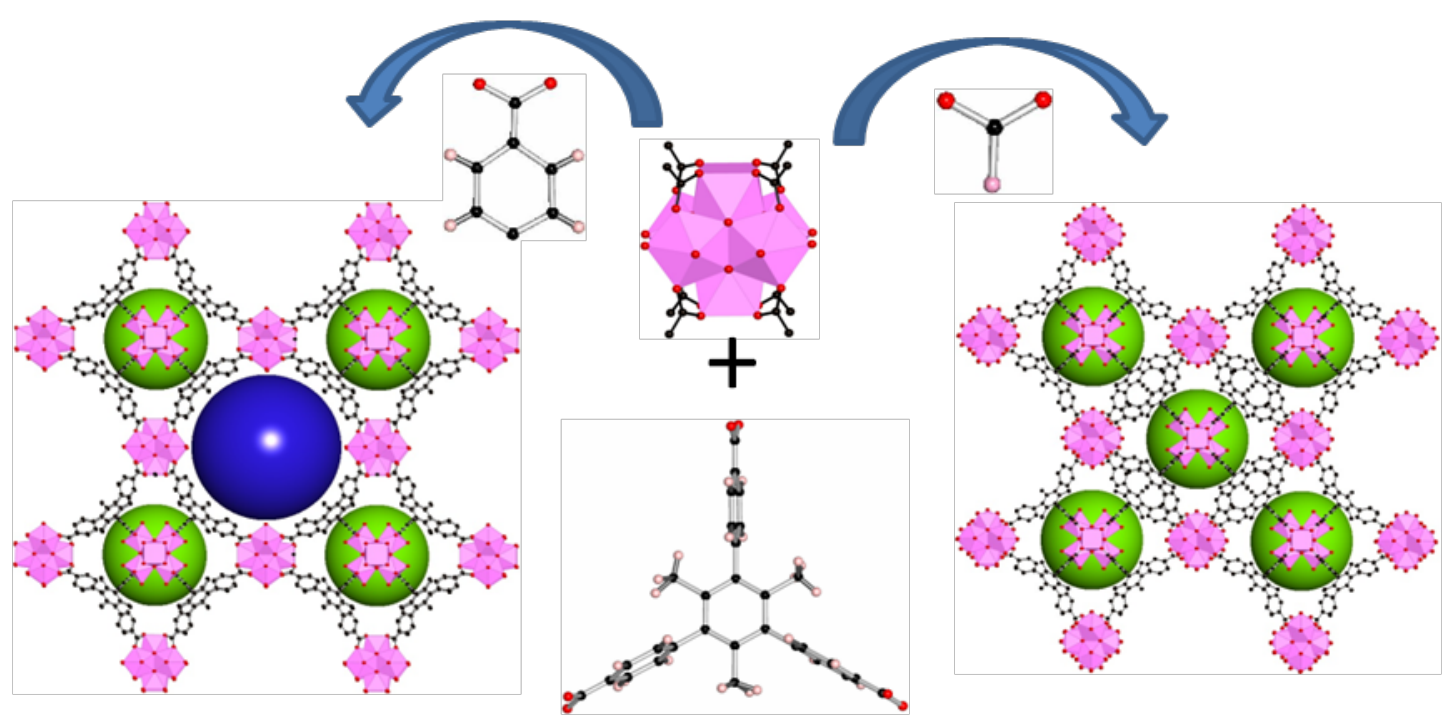

